\title{
The NJL-jet model for quark fragmentation functions
}

\author{
T. Ito, W. Bentz \\ Department of Physics, School of Science, Tokai University, \\ Hiratsuka-shi, Kanagawa 259-1292, Japan \\ I.C. Cloët \\ Department of Physics, University of Washongton, \\ Seattle, WA 98195-1560, USA \\ A.W. Thomas \\ Jefferson Lab, 12000 Jefferson Avenue, \\ Newport News, VA 23606, USA \\ K. Yazaki \\ Radiation Laboratory, Nishina Accelerator Research Center, \\ RIKEN, Wako, Saitama 351-0198, Japan
}

*Correspondence to: T. Ito, e-mail: 8atad002@mail.tokai-u.jp 


\begin{abstract}
A description of fragmentation functions which satisfy the momentum and isospin sum rules is presented in an effective quark theory. Concentrating on the pion fragmentation function, we first explain the reason why the elementary (lowest order) fragmentation process $q \rightarrow q \pi$ is completely inadequate to describe the empirical data, although the "crossed" process $\pi \rightarrow q \bar{q}$ describes the quark distribution functions in the pion reasonably well. Then, taking into account cascade-like processes in a modified jet-model approach, we show that the momentum and isospin sum rules can be satisfied naturally without introducing any ad-hoc parameters. We present numerical results for the Nambu-Jona-Lasinio model in the invariant mass regularization scheme, and compare the results with the empirical parametrizations. We argue that this NJL-jet model provides a very useful framework to calculate the fragmentation functions in an effective chiral quark theory.
\end{abstract}




\section{Introduction}

Quark distribution and fragmentation functions are the basic nonperturbative ingredients for a QCD-based analysis of hard scattering processes [1][6]. Distribution functions can be extracted by analyzing inclusive processes $[7,8]$, and their description in terms of effective quark theories of QCD has been quite successful [9]. In recent years, a lot of effort was made to extract the fragmentation functions by analyzing inclusive hadron production (semiinclusive) processes in $\left(e^{+}, e^{-}\right)$annihilation, deep-inelastic lepton-nucleon scattering, and proton-proton collisions[10]. Besides being of fundamental interest in their own right, the knowledge of fragmentation functions is very important to extract the transversities $[11,6]$ from the data, and to analyse several interesting effects in semi-inclusive processes[12].

Because of the importance of the fragmentation functions, many attempts have been made to describe them in effective quark theories [13]. However, in order to achieve a reasonable agreement with the empirical parametrizations, it was necessary to introduce new parameters, like normalization constants, which cannot not be justified on theoretical grounds. A description of fragmentation functions in effective quark theories, which naturally satisfy the relevant sum rules [3] and describe the empirical data reasonably well without introducing new parameters into the theory, has not yet been achieved.

This failure to describe the fragmentation functions in the same framework which was successful for the distribution functions, is surprising, because there exists a general relation, the so called Drell-Levy-Yan (DLY) relation $[14,15]$, which suggests a way to compute the fragmentation functions by analytic continuation of the distribution functions into the region of Bjorken $x>1$. Although the derivation of this relation appears to be 
very general (as we show in Appendix A of this paper), the basic assumption that the distributions and fragmentations are essentially one and the same function, defined in different regions of the variable, has not been proved. Moreover, the approximations used to calculate the distribution functions may not be justified for the fragmentation functions, and vice versa. For example, in the fragmentation process of a quark into a pion, $q \rightarrow \pi+n$, where $n$ is a spectator, there is no a priori reason to truncate $n$ to a single quark state, as the DLY crossing arguments would suggest for the case of a Bethe-Salpeter type vertex function for $\pi \rightarrow q \bar{q}$. One can actually give a quantitative argument [16] that the lightest component of $n$ is dominant only if the scaling variable $z$ is very close to 1 .

On the other hand, the phenomenological quark jet-model, as formulated originally by Field and Feynman [17], suggests that the meson observed in a semi-inclusive process is one among many others, i.e., the spectator state $n$ contains a lot of mesons. This model is based on a "product ansatz" for a chain of elementary fragmentation process, where in each step a certain fraction of the quark momentum is transfered to a meson, until eventually a very soft quark remains, which is assumed to annihilate with the other remnants of the process without producing further observed mesons ${ }^{1}$. In order that $100 \%$ of the quark light-cone momentum is transferred to the mesons, it is actually necessary to assume an infinite number of steps (mesons) in the decay chain, as will be explained in more detail in Sect. 4 of this paper. In this case, it is possible to satisfy the momentum sum rule for fragmentation functions [3], as it is supposed to be valid in QCD-based fits to the data

\footnotetext{
${ }^{1}$ This picture of independent fragmentation is appealing because of its simplicity. More elaborate models for hadronization are the string model [18] or the cluster model [19], which are suitable for Monte Carlo analysis.
} 
[10]. Clearly, this sum rule cannot be satisfied in a single step elementary fragmentation process.

The purpose of this paper is to apply the method of the quark jet-model to calculate the spin-independent fragmentation functions in an effective chiral quark theory, which proved to be very successful for the description of quark distribution functions. We will concentrate on the quark fragmentation into pions in the Nambu-Jona-Lasinio (NJL) model [20], but the methods shown here can easily be extended to other fragmentation channels, and applied to other effective quark theories. In order to reconcile the quark jet-model with our present NJL model description, we will introduce an extended product ansatz, which allows the fragmentation of a quark into a finite number of pions according to a certain distribution function, and in the end take the limit of infinitely many pions. We will show how the momentum and isospin sum rules emerge naturally without introducing any new parameters into the theory. Our numerical results will show that this NJL-jet model provides a very reasonable framework to describe the fragmentation functions.

This paper is organized as follows: In Sect.2 we start from the operator definitions to discuss the sum rules and the DLY relation. In Sect.3 we give the expressions for the elementary fragmentation functions in the NJL model, and discuss their physical interpretations and sum rules. In Sect.4 we introduce the extended product ansatz to describe a chain of elementary fragmentation processes in the spirit of the quark jet-model, derive the integral equation for the fragmentation function, and discuss the momentum and isospin sum rules. In Sect.5 we explain the model framework for the numerical calculations, show the results and compare them with the empirical fragmentation functions. A summary will be given in Sect.6. 


\section{Operator definitions and sum rules}

Operator definitions and sum rules for fragmentation functions have been first given in Ref.([3]) (see also Ref.([21])). Here we summarize the basic relations, together with those for the distribution functions for clarity.

The spin-independent distribution function of a quark (flavor $q$ ) inside a hadron (spin-flavor $h$, for example $h=p \uparrow, \pi^{+}$, etc.), and the spinindependent fragmentation function for $q \rightarrow h$ are defined by

$$
\begin{aligned}
f_{q}^{h}(x) & =\frac{1}{2} \int \frac{\mathrm{d} \omega^{-}}{2 \pi} e^{i p_{-} \omega^{-} x} \hat{\sum}_{n}\left\langle p(h)|\bar{\psi}| p_{n}\right\rangle \gamma^{+}\left\langle p_{n}\left|\psi\left(\omega^{-}\right)\right| p(h)\right\rangle \\
D_{q}^{h}(z) & =\frac{z}{12} \int \frac{\mathrm{d} \omega^{-}}{2 \pi} e^{i p_{-} \omega^{-} / z} \hat{\sum}_{n}\left\langle p(h), p_{n}|\bar{\psi}| 0\right\rangle \gamma^{+}\left\langle 0\left|\psi\left(\omega^{-}\right)\right| p(h), p_{n}\right\rangle
\end{aligned}
$$

Here the field operators $\psi$ (and also $b, b^{\dagger}$ below) refer to the quark flavor $q$, although it is not indicated explicitly. The symbol $p(h)$ means a hadron $h$ with momentum $p$, and $p_{n}$ refers to the spectator state. The summation symbol $\hat{\sum}_{n}$ includes an integration over the on-shell momenta $p_{n}$. The light cone components of a 4 -vector are defined as $a^{\mu}=\left(a^{+}, a^{-}, \boldsymbol{a}_{T}\right)$ with $a^{ \pm}=$ $\left(a^{0} \pm a^{3}\right) / \sqrt{2}$. Both expressions (2.1) and (2.2) refer to a frame where $\boldsymbol{p}_{T}=$ 0 . Covariant normalization is used throughout this paper ${ }^{2}$. The physical content of the functions (2.1) and (2.2) is most transparent if we introduce the "good" light cone quark field $\psi_{+}[22]-[24]$, using $\bar{\psi} \gamma^{+} \psi=\sqrt{2} \psi_{+}^{\dagger} \psi_{+}$, and the expansion of $\psi_{+}$given in footnote 2 . This gives the following relations,

\footnotetext{
${ }^{2}$ In this normalization, $<p\left(h^{\prime}\right) \mid p(h)>=2 V p_{-} \delta_{h h^{\prime}}(V$ is the volume of the system), and $\left|p(h), p_{n}\right\rangle=\sqrt{2(2 \pi)^{3} p_{-}} a_{h}^{\dagger}(p)\left|p_{n}\right\rangle$ with $\left[a_{h}\left(p^{\prime}\right), a_{h}(p)\right]_{ \pm}=\delta^{(3)}\left(\boldsymbol{p}^{\prime}-\boldsymbol{p}\right)$. The expansion of $\psi_{+} \equiv \Lambda_{+} \psi$ (where $\Lambda_{+}=\frac{1}{2} \gamma^{-} \gamma^{+}$) reads ( $\alpha$ denotes spin and color)

$$
\psi_{+}\left(\omega^{-}\right)=\int_{0}^{\infty} \frac{\mathrm{d} k_{-}}{\sqrt{2 k_{-}}} \int \frac{\mathrm{d}^{2} k_{T}}{(2 \pi)^{3 / 2}} \sum_{\alpha}\left(b_{\alpha}(k) u_{\alpha}(k) e^{-i k_{-} \omega^{-}}+d_{\alpha}^{\dagger}(k) v_{\alpha}(k) e^{i k_{-} \omega^{-}}\right) .
$$
}


which are independent of the normalization of states:

$$
\begin{aligned}
f_{q}^{h}(x) \mathrm{d} x & =\mathrm{d} k_{-} \int \mathrm{d}^{2} k_{T} \sum_{\alpha} \frac{\left\langle p(h)\left|b_{\alpha}^{\dagger}(k) b_{\alpha}(k)\right| p(h)\right\rangle}{\langle p(h) \mid p(h)\rangle}, \\
D_{q}^{h}(z) \mathrm{d} z & =\frac{z^{2}}{6} \mathrm{~d} p_{-} \int \mathrm{d}^{2} k_{T} \sum_{\alpha} \frac{\left\langle k(\alpha)\left|a_{h}^{\dagger}(p) a_{h}(p)\right| k(\alpha)\right\rangle}{\langle k(\alpha) \mid k(\alpha)\rangle} .
\end{aligned}
$$

Here $\mathrm{d} x=\mathrm{d} k_{-} / p_{-}$(that is, $k_{-}=p_{-} x$ for some fixed $\left.p_{-}>0\right)$, and $\mathrm{d} z=$ $\mathrm{d} p_{-} / k_{-}$(that is, $p_{-}=k_{-} z$ for some fixed $k_{-}>0$ ). The symbol $\alpha$ denotes the spin-color of the quark with flavor $q$.

According to Eq.(2.3), we can interpret $f_{q}^{h}(x)$ as the light cone momentum distribution of $q$ in $h$, where a sum over spin-color of $q$ is understood, while the spin of $h$ is fixed (although the result is independent of this spin direction, since we consider only the spin independent distributions here). As noted above, Eq.(2.4) refers to the frame where the produced $h$ has $\mathbf{p}_{T}=0$ but the fragmenting quark has non-zero $\mathbf{k}_{T}$. To interpret the result as a distribution of $h$ in $q$, one has to make a Lorentz transformation to the frame where $\mathbf{k}_{\perp}=0$ but $h$ has non-zero $\mathbf{p}_{\perp}$. (Note the distinction between the symbols $T$ and $\perp$.) This is discussed in detail in Refs. [3, 6], and as a result one can simply substitute

$$
\boldsymbol{k}_{T}=-\frac{\boldsymbol{p}_{\perp}}{z}
$$

leaving all other things unchanged. Then we obtained from (2.4)

$$
D_{q}^{h}(z) \mathrm{d} z=\frac{1}{6} \mathrm{~d} p_{-} \int \mathrm{d}^{2} p_{\perp} \sum_{\alpha} \frac{\left\langle k(\alpha)\left|a_{h}^{\dagger}(p) a_{h}(p)\right| k(\alpha)\right\rangle}{\langle k(\alpha) \mid k(\alpha)\rangle},
$$

where now the fragmenting quark has $\boldsymbol{k}_{\perp}=0$. According to (2.6), we can interpret $D_{q}^{h}(z)$ as the light cone momentum distribution of $h$ in $q$, where the factor $1 / 6$ indicates an average [21] of the spin-color of $q$, while the spin 
of $h$ is fixed ${ }^{3}$. In fact, for the elementary distribution and fragmentation functions considered in the next section, the naively expected relation

$$
D_{q}^{h}(z)=\frac{f_{h}^{q}(z)}{d_{h}}
$$

is valid. Here $d_{h}$ is the spin degeneracy (or, in the generalized case, the spincolor degeneracy) of $h$. Generally, however, this relation is not necessarily valid, because $q$ is off-shell (its virtuality being determined kinematically by the scaling variable and the transverse momentum) and $h$ is on-shell, which breaks the naive symmetry under the interchange $q \leftrightarrow h$.

To get the momentum sum rule from Eq. (2.6), we multiply both sides by $z=p_{-} / k_{-}$, integrate over $z$ from 0 to 1 , and sum over $h^{4}$. Then one observes that the momentum operator represented in terms of hadron operators is

$$
\hat{P}_{-} \equiv \sum_{h} \int_{0}^{\infty} \mathrm{d} p_{-} \int \mathrm{d}^{2} p_{\perp}\left(p_{-} a_{h}^{\dagger}(p) a_{h}(p)\right)
$$

By assuming that the quark state $\mid k(\alpha)>$ in (2.6) is an eigenstate of this operator with eigenvalue $k_{-}$, we obtain the momentum sum rule

$$
\sum_{h} \int_{0}^{1} z \mathrm{~d} z D_{q}^{h}(z)=1
$$

The physical content of (2.9) is that $100 \%$ of the inital quark light cone momentum $\left(k_{-}\right)$is transfered to the hadrons. The condition which lies at

\footnotetext{
${ }^{3}$ For the generalized case, where $h$ can also be a quark, we summarize the definitions as follows: $f_{q}^{h}(x)$ refers to fixed flavors $q, h$. All other quantum numbers of $q$ (spin, color, etc) are summed over, while those of $h$ are fixed. $D_{q}^{h}(z)$ refers also to fixed flavors $q, h$. But there is an average over the other quantum numbers of $q$ (spin, color, etc), while those of $h$ are fixed. This definition has the advantage that in a semi-inclusive process, which involves the product $f_{q}^{T}(x) D_{q}^{h}(z)$, the quark spin-color summation is included in $f$ but not in $D$, which avoids double counting.

${ }^{4} \mathrm{~A}$ subtle point here is that, in order to get an integral $\int_{0}^{\infty} \mathrm{d} p_{-}$on the r.h.s. of (2.9), one has to chose $k_{-}=\infty$. This does not influence the result, which depends only on $z$.
} 
the basis of (2.9) is that the initial quark state is an eigenstate of the momentum operator (2.8) expressed solely in terms of hadrons, i.e; that the quark hadronizes completely in the sense that it gives all of its light cone momentum to the hadrons.

A similar argument leads to the isospin sum rule [3]

$$
\sum_{h} \int_{0}^{1} \mathrm{~d} z t_{h} D_{q}^{h}(z)=t_{q}
$$

where $t_{q}$ and $t_{h}$ denote the 3 -components of the isospins of $q$ and $h$. The physical content of this sum rule is the $100 \%$ is the isospin of the initial quark is transfered to hardons, which is possible only if we average over the isospin of the soft quark remainder of a fragmentation chain (see Sect.4). In general, there is no sum rule for the baryon number or electric charge, because the baryon number or average electric charge of the quark remainder is not zero ${ }^{5}$. If we simply integrate both sides of (2.6) over $z$, we get the hadron multiplicity, which can be interpreted as the "number of mesons per quark". There is, however, no conservation law which leads to a sum rule for the multiplicity.

There is an interesting relation, based on charge conjugation and crossing symmetry, between the fragmentation function for physical $z<1$ and the distribution function for unphysical $x>1$ :

$$
D_{q}^{h}(z)=(-1)^{2\left(s_{q}+s_{h}\right)+1} \frac{z}{d_{q}} f_{q}^{h}\left(x=\frac{1}{z}\right)
$$

which is called the Drell-Levy-Yan relation $[14,15]$. Here $s_{q}\left(s_{h}\right)$ and $d_{q}$ are the spins of $q(h)$ and the spin-color degeneracy of $q$. We derive this relation in Appendix A in two independent ways: The first method, which follows the original arguments [14], compares the hadronic tensors for $e h \rightarrow e^{\prime} X$

\footnotetext{
${ }^{5}$ The average of the electric charge is zero only if one imposes flavor SU(3) symmetry.
} 
(inclusive DIS) and $e^{+} e^{-} \rightarrow h X$ (inclusive hadron production), and uses crossing relations for matrix elements of the current operator. The second method - which to the best of our knowledge has not been worked out before - starts directly from the operator definitions (2.1) and (2.2), and uses charge conjugation and crossing symmetries for matrix elements of the quark field operator.

If one has an effective quark theory to calculate the quark distribution functions, relation (2.11) would suggest a straight forward way to obtain the fragmentation functions. As will become clear in the following sections for the lowest order (elementary) processes, however, such an attempt leads to disastrous results: The fragmentation functions obtained in this way are one or two magnitudes smaller than the empirical functions, and the sum rules (2.9) and (2.10) are not valid.

The reasons why relation (2.11) fails in actual applications are as follows: (i) It is based on the assumption that the distribution functions can be continued analytically beyond $x=1$. However, it is well known that the $Q^{2}$ evolution equations lead to singularities at $x=1$, which are (regularized) infrared singularities arising from the vanishing gluon mass [5, 25] and render an analytic continuation impossible. Still one may argue that (2.11) should be used only at the low energy (model) scale, but actually it is broken also there because of the cut-off regularization, as we will discuss in detail in the next section. (ii) Most importantly, approximations which work reasonably well for the distribution functions need not be valid for the fragmentation functions, and vice versa. For example, the assumption that the pion is a $q \bar{q}$ Bethe-Salpeter bound state is very reasonable for the distribution function [9], but the DLY crossing arguments then imply the truncation of the specta- 
tor state $p_{n}$ of (2.2) to a single quark state. Although this simple assumption does not lead to any violation of conservation laws, the sum rules (2.9) and (2.10) cannot be satisfied in a single step fragmentation process.

Because of these reasons, we will not rely on Eq.(2.11) to calculate the fragmentation functions, although we will confirm its formal validity for the lowest order (elementary) functions. We note the above arguments do not question the usefulness of Eq.(2.11) as a means to relate the kernels of the $Q^{2}$ evolution equations for distributions and fragmentations (see Ref.[15] and Appendix B). In fact, it is known that in leading order (LO) of $\alpha_{s}$ this relation between the kernels is valid, although it is violated in next to leading order (NLO) [26].

\section{Elementary distribution and fragmentation functions}

The elementary distribution and fragmentation functions for the case of the pion are represented in Fig.1 as cut diagrams. Because it is well known that the distribution function can also be obtained by a straight forward Feynman diagram calculation $[27,24]^{6}$, we also present the Feynman diagram in Fig.1a. We obtain the following expressions ${ }^{7}$ :

$$
\begin{aligned}
f_{q}^{\pi}(x) & =\frac{1}{2}\left(1+\tau_{\pi} \tau_{q}\right) 3 g_{\pi}^{2} \int \frac{\mathrm{d}^{4} k}{(2 \pi)^{4}} \operatorname{Tr}_{D}\left[S_{F}(k) \gamma^{+} S_{F}(k) \gamma_{5}(\not k-\not p-M) \gamma_{5}\right] \\
& \times \delta\left(k_{-}-p_{-} x\right) \delta\left((p-k)^{2}-M^{2}\right)
\end{aligned}
$$

\footnotetext{
${ }^{6}$ This is simply seen by using completeness in Eq.(2.1) and the identity $\psi_{+}(0)^{\dagger} \psi_{+}\left(\omega^{-}\right)=T\left(\psi_{+}(0)^{\dagger} \psi_{+}\left(\omega^{-}\right)\right)$in the limit $\omega^{+} \rightarrow 0-\epsilon$, which follows from causality.

${ }^{7}$ The expressions given in this section refer to the NJL model, but actually they have the same form in any effective chiral quark model. We denote the elementary fragmentation function by $d$ in order to distinguish it from the total fragmentation function $D$ determined in Sect. 4.
} 

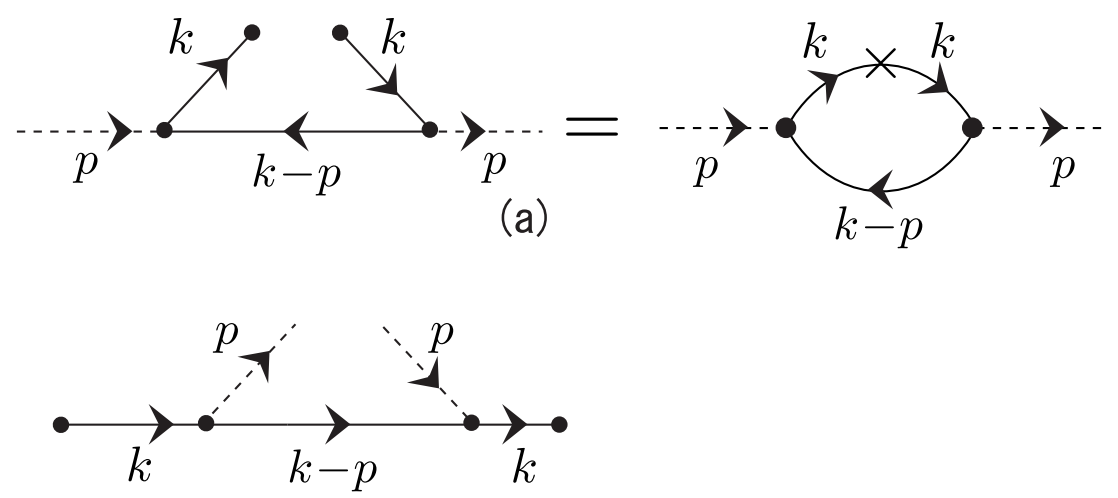

(b)

Figure 1: (a) Cut diagram (left) and Feynman diagram (right) for the distribution function $f_{q}^{\pi}(x)$. Solid lines denote the quark, and dashed lines the pion. Here $k_{-}=p_{-} x$, and the two quark lines with momentum $k$ are connected by $\gamma^{+}$. (b) Cut diagram for the fragmentation function $d_{q}^{\pi}(z)$. Here $p_{-}=k_{-} z$, and the two quark lines with momentum $k$ are connected by $\gamma^{+}$. The diagram refers to the frame $\boldsymbol{p}_{T}=0$, and the substitution (2.5) is performed in the final transverse momentum integral.

$$
\begin{aligned}
& =\frac{1}{2}\left(1+\tau_{\pi} \tau_{q}\right) 6 g_{\pi}^{2} \int \frac{\mathrm{d}^{2} k_{T}}{(2 \pi)^{3}} \frac{\boldsymbol{k}_{T}^{2}+M^{2}}{\left[\boldsymbol{k}_{T}^{2}+M^{2}-M_{\pi}^{2} x(1-x)\right]^{2}} \\
d_{q}^{\pi}(z) & =\frac{1}{2}\left(1+\tau_{\pi} \tau_{q}\right) \frac{z}{2} g_{\pi}^{2} \int \frac{\mathrm{d}^{4} k}{(2 \pi)^{4}} \operatorname{Tr}_{D}\left[S_{F}(k) \gamma^{+} S_{F}(k) \gamma_{5}(\not k-\not p-M) \gamma_{5}\right] \\
& \times \delta\left(k_{-}-p_{-} / z\right) \delta\left((p-k)^{2}-M^{2}\right) \quad\left(=\frac{z}{6} f_{q}^{\pi}\left(x=\frac{1}{z}\right)\right) \\
& =\frac{1}{2}\left(1+\tau_{\pi} \tau_{q}\right) g_{\pi}^{2} z \int \frac{\mathrm{d}^{2} p_{\perp}}{(2 \pi)^{3}} \frac{\boldsymbol{p}_{\perp}^{2}+M^{2} z^{2}}{\left[\boldsymbol{p}_{\perp}^{2}+M^{2} z^{2}+(1-z) M_{\pi}^{2}\right]^{2}}
\end{aligned}
$$

Here $S_{F}$ is the Feynman propagator of the constituent quark (mass $M$ ), and the square of the quark-pion coupling constant $g_{\pi}$ in the NJL model is defined as the residue of the $q \bar{q}$ t-matrix at the pion pole. It can be expressed in terms of the $q \bar{q}$ bubble graph $\Pi_{\pi}\left(q^{2}\right)=6 i \int \frac{\mathrm{d}^{4} k}{(2 \pi)^{4}} \operatorname{Tr}_{D}\left[\gamma_{5} S_{F}(k) \gamma_{5} S_{F}(k+q)\right]$ by

$$
g_{\pi}^{2}=-\left(\partial \Pi_{\pi}\left(q^{2}\right) / \partial q^{2}\right)_{q^{2}=M_{\pi}^{2}}^{-1} .
$$


We use the isospin notations $\left(\tau_{u}, \tau_{d}\right)=(1,-1), \quad\left(\tau_{\pi^{+}}, \tau_{\pi^{0}}, \tau_{\pi^{-}}\right)=(1,0,-1)$. For the distribution function in the physical region $(x<1)$, a factor $\Theta\left(p_{-}-k_{-}\right)=$ $\Theta(1-x)$ has to be supplied in (3.1), which expresses the fact that the intermediate antiquark in Fig.1a has positive energy. Similarly, for the fragmentation function a factor $\Theta\left(k_{-}-p_{-}\right)=\Theta(1-z)$ has to be supplied in (3.3), because the intermediate quark in Fig.1b has positive energy. To get (3.4) we made the substitution (2.5).

The DLY relation on this level, as indicated in brackets in (3.3), shows that Eq.(3.1) can be considered as a generalized distribution function, which gives the physical distribution function in the region $x<1$ and the fragmentation function in the region $x=1 / z>1$. The reason why we indicate this relation only in brackets is that it is violated if the integrals are regularized: For example, if we use a sharp cut-off $(\Lambda)$ for the transverse quark momentum in (3.2), a strict application of the DLY relation would mean that the transverse momentum of the produced pion in (3.4) should be cut at $\Lambda z$, which is unacceptable. The more physical procedure is to impose $\left|\boldsymbol{k}_{T}\right|<\Lambda$ on (3.2) and $\left|\boldsymbol{p}_{\perp}\right|<\Lambda$ on (3.4), which breaks the DLY relation. A similar breakdown of the DLY relation occurs in any other sensible regularization scheme. An noticeable consequence of this is that in the chiral limit the distribution function (3.2) becomes a constant, but the fragmentation function (3.4) is not linear in $z$, as the DLY relation indicated in (3.3) would suggest.

The relations for the distribution function

$$
\int_{0}^{1} \mathrm{~d} x f_{q}^{\pi}(x)=\frac{1}{2}\left(1+\tau_{\pi} \tau_{q}\right), \quad \int_{0}^{1} x \mathrm{~d} x f_{q}^{\pi}(x)=\frac{1}{2}\left(1+\tau_{\pi} \tau_{q}\right) \cdot \frac{1}{2},
$$

lead to the usual number and momentum sum rules. For the fragmentation 
function, one discovers the following relation from (3.4):

$$
\int_{0}^{1} \mathrm{~d} z d_{q}^{\pi}(z)=\frac{1}{3}\left(1+\tau_{\pi} \tau_{q}\right)\left(1-Z_{Q}\right) \Rightarrow \int_{0}^{1} \mathrm{~d} z \sum_{\tau_{\pi}} d_{q}^{\pi}(z)=1-Z_{Q} .
$$

Here $Z_{Q}$ is the residue of the quark propagator in the presence of the pion cloud, and is expressed in terms of the renormalized quark self energy $\Sigma_{Q}^{(\pi)}(k)$ of Fig.2 as

$$
\begin{aligned}
1-Z_{Q} & =-\left(\frac{\partial \Sigma_{Q}^{(\pi)}}{\partial \not k}\right)_{\not k=M}=-\frac{M}{k_{-}}\left(\bar{u}_{Q}(k) \frac{\partial \Sigma_{Q}^{(\pi)}}{\partial k_{+}} u_{Q}(k)\right) \\
& =\frac{3}{2} g_{\pi}^{2} \int_{0}^{1} z \mathrm{~d} z \int \frac{\mathrm{d}^{2} p_{\perp}}{(2 \pi)^{3}} \frac{\boldsymbol{p}_{\perp}^{2}+M^{2} z^{2}}{\left[\boldsymbol{p}_{\perp}^{2}+M^{2} z^{2}+(1-z) M_{\pi}^{2}\right]^{2}},
\end{aligned}
$$

where $u_{Q}$ is the quark spinor $\left(\bar{u}_{Q} u_{Q}=1\right)$. Because $Z_{Q}$ is interpreted as

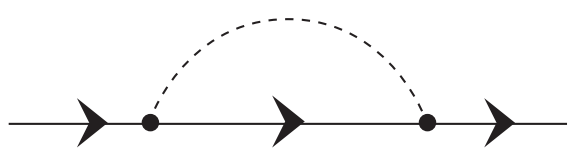

Figure 2: The quark self energy, $\Sigma_{Q}^{(\pi)}(k)=-3 i g_{\pi}^{2} \int \frac{\mathrm{d}^{4} p}{(2 \pi)^{4}} \gamma_{5} S_{F}(k-p) \gamma_{5} \Delta_{F}(p)$, where $\Delta_{F}$ is the Feynman propagator of the pion.

the probability to find a "bare" constituent quark without the pion cloud, relation (3.7) indicates that the elementary fragmentation function is normalized to the "number of pions per quark". This is expected from our general discussions in Sect.2, and will be further elucidated below. Because typical values of $Z_{Q}$ in models based on constituent quarks are between 0.8 and 0.9 , we see that the momentum sum rule $\int_{0}^{1} z \mathrm{~d} z \sum_{\tau_{\pi}} d_{q}^{\pi}(z)$ will be much smaller than the typical empirical values (for example $\simeq 0.74$ in the NLO analysis of the first paper in Ref.[10]). From this we can anticipate that the elementary fragmentation functions will be very small compared to the empirical ones (see Sect. 5). 
In order to confirm that this does not mean that momentum conservation is violated, we also give the expressions for the distribution function of a quark $(q)$ inside a parent quark $(Q)$, and for the fragmentation function of $q$ to $Q$. The relevant cut diagrams are shown in Fig. 3, and a straight forward calculation, following the rules indicated already in Eqs.(3.1) and (3.3), gives

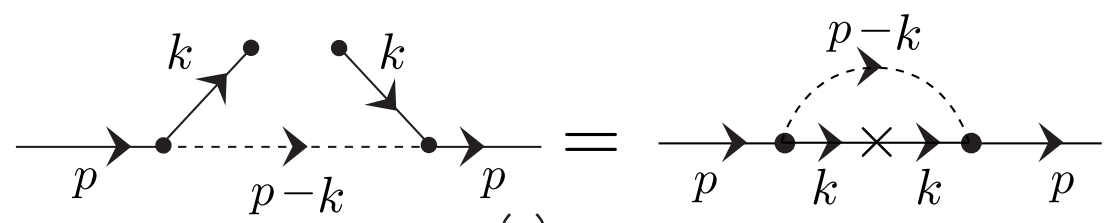

(a)

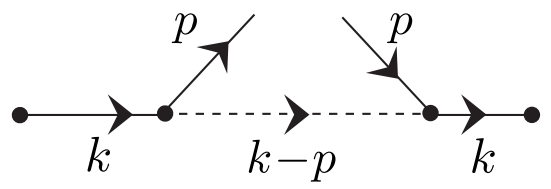

(b)

Figure 3: (a) Cut diagram (left) and Feynman diagram (right) for the loop term in $f_{q}^{Q}(x)$ of Eq.(3.9). Here $k_{-}=p_{-} x$, and the two quark lines with momentum $k$ are connected by $\gamma^{+}$. (b) Cut diagram for the loop term in $d_{q}^{Q}(z)$ of Eq.(3.10). Here $p_{-}=k_{-} z$, and the two quark lines with momentum $k$ are connected by $\gamma^{+}$. The diagram refers to the frame $\boldsymbol{p}_{T}=0$, and the substitution (2.5) is performed in the final transverse momentum integral.

$$
f_{q}^{Q}(x)=Z_{Q} \delta(x-1) \delta_{q, Q}+\left(\frac{1}{2}-\frac{\tau_{q} \tau_{Q}}{6}\right)
$$

\footnotetext{
${ }^{8}$ The tree level terms $\propto Z_{Q}$ in $(3.9)$ and (3.10) come from the vacuum state in the sum over $n$ in (2.1) and (2.2), which contributes for the case where $p(h)$ is a quark. Using $\psi=\sqrt{Z_{Q}} \hat{\psi}$, where $\hat{\psi}$ is the renormalized quark field with unit pole residue of the propagator, gives the $Z_{Q}$-terms in (3.9) and (3.10). (In the loop terms, all factors $Z_{Q}$ of the propagators cancel each other.) We also note that the loop terms in $f_{q}^{Q}(x)$ and $d_{q}^{Q}(z)$ formally satisfy the DLY relation $d_{q, \text { loop }}^{Q}(z)=(-z / 6) f_{q, \text { loop }}^{Q}(x=1 / z)$, but it is violated after regularization.
} 


$$
\begin{aligned}
& \times \frac{3}{2} g_{\pi}^{2}(1-x) \int \frac{\mathrm{d}^{2} k_{T}}{(2 \pi)^{3}} \frac{\boldsymbol{k}_{T}^{2}+M^{2}(1-x)^{2}}{\left[\boldsymbol{k}_{T}^{2}+M^{2}(1-x)^{2}+x M_{\pi}^{2}\right]^{2}}, \\
d_{q}^{Q}(z) & =\frac{1}{6} Z_{Q} \delta(z-1) \delta_{q, Q}+\frac{1}{6}\left(\frac{1}{2}-\frac{\tau_{q} \tau_{Q}}{6}\right) \\
& \times \frac{3}{2} g_{\pi}^{2}(1-z) \int \frac{\mathrm{d}^{2} p_{\perp}}{(2 \pi)^{3}} \frac{\boldsymbol{p}_{\perp}^{2}+M^{2}(1-z)^{2}}{\left[\boldsymbol{p}_{\perp}^{2}+M^{2}(1-z)^{2}+z M_{\pi}^{2}\right]^{2}} .
\end{aligned}
$$

These relations show that

$$
d_{q}^{Q}(z)=\frac{1}{6} f_{q}^{Q}(z)=\frac{1}{6} f_{Q}^{q}(z)
$$

in accordance with (2.7), and therefore the two quantities (3.9) and (3.10) describe essentially the same object, namely the splitting function of a quark to another quark, which also includes a "non-splitting" term $\propto Z_{Q}$. The normalization is

$$
\int_{0}^{1} \mathrm{~d} z 6 \sum_{\tau_{Q}} d_{q}^{Q}(z)=Z_{Q}+\left(1-Z_{Q}\right)=1
$$

where the factor 6 represents the summation over spin and color of $Q$.

As is physically expected, the second term in (3.10) can be obtained from the $q \rightarrow \pi$ fragmentation function (3.4) by the substitutions $z \rightarrow 1-z$ and $\tau_{\pi} \rightarrow \tau_{q} / 2-\tau_{Q} / 2$, and this directly leads to the momentum conservation for the fragmentation of $q$ into either $Q$ or $\pi$ (see Eq.(3.15) below).

This connection between splitting functions can also be viewed also in another way: The second term in (3.9), which describes the distribution of $q$ inside $Q$ with a pion spectator, suggests that by the substitutions $\tau_{q} / 2 \rightarrow$ $\tau_{Q} / 2-\tau_{\pi}$ and $x \rightarrow 1-x$ we obtain the distribution function of a pion inside the quark $Q$ :

$$
f_{\pi}^{Q}(x)=\frac{1}{2}\left(1+\tau_{\pi} \tau_{Q}\right) g_{\pi}^{2} x \int \frac{\mathrm{d}^{2} k_{T}}{(2 \pi)^{3}} \frac{\boldsymbol{k}_{T}^{2}+M^{2} x^{2}}{\left[\boldsymbol{k}_{T}^{2}+M^{2} x^{2}+(1-x) M_{\pi}^{2}\right]^{2}},
$$


and comparison to (3.4) gives $d_{q}^{\pi}(z)=f_{\pi}^{q}(z)$, again in accordance with (2.7). This relation further elucidates the interpretation of the normalization (3.7) as the number of pions per quark:

$$
\int_{0}^{1} \mathrm{~d} z \sum_{\tau_{\pi}} d_{q}^{\pi}(z)=\int_{0}^{1} \mathrm{~d} z \sum_{\tau_{\pi}} f_{\pi}^{q}(z)=1-Z_{Q} .
$$

We finally write down the momentum sum rules for the elementary splitting functions. In terms of the distribution functions we have

$$
\begin{array}{rl}
\int_{0}^{1} & x \mathrm{~d} x\left(\sum_{\tau_{q}} f_{q}^{Q}(x)+\sum_{\tau_{\pi}} f_{\pi}^{Q}(x)\right) \\
=Z_{Q}+\int_{0}^{1} x \mathrm{~d} x \sum_{\tau_{\pi}} f_{\pi}^{Q}(1-x)+\int_{0}^{1} x \mathrm{~d} x \sum_{\tau_{\pi}} f_{\pi}^{Q}(x)=1,
\end{array}
$$

where in the second equality we used $x \rightarrow 1-x$ and Eq.(3.13). In terms of the fragmentation functions, this becomes

$$
\int_{0}^{1} z \mathrm{~d} z\left(6 \sum_{\tau_{Q}} d_{q}^{Q}(z)+\sum_{\tau_{\pi}} d_{q}^{\pi}(z)\right)=1 .
$$

Referring here to the form (3.10), we have the following simple interpretation of the momentum sum rule (3.15): Because $Z_{Q}$ is the probability that the initial quark $q$ does not fragment at all, the fraction $Z_{Q}$ of the momentum stays with the initial quark. The remaining fraction $\left(1-Z_{Q}\right)$ is shared among the quark remainder (first term in (3.15)) and the produced pion (second term in $(3.15))$.

To conclude, although a description of fragmentation functions by using only the elementary fragmentation processes does not violate any conservation laws, it is completely inadequate because of the following reasons: First, there is a large probability $\left(Z_{Q}\right)$ that the initial quark does not fragment at all. Second, if it fragments, the momentum fraction $1-Z_{Q}$ is shared between the quark remainder and the pion. Both points are in contradiction to 
the usual assumption of complete hadronization, which is expressed by the momentum sum rule (2.9).

\section{Product ansatz for quark cascades}

From the previous section, it is clear that we have to consider the possibility that the fragmenting quark produces a cascade of mesons. A simple model to describe cascades is the quark jet model of Field and Feynman [17]. The product ansatz used in [17], however, assumes from the outset that the probability for fragmentation in each elementary process is $100 \%$, and that the quark produces a very large number (actually an infinite number) of mesons. Because these assumptions are inconsistent with our present effective quark theory, we will first introduce a modified product ansatz, and then explain its physical significance and its relation to the original quark jet model.

We assume that the maximum number of mesons which can be produced by the fragmenting quark is $N$. We then consider a process where the initial quark with light cone momentum $k_{-} \equiv W_{0}$ (which we will simply call the "momentum" in the following) goes through a sequence of momenta $W_{0} \geq$ $W_{1} \geq W_{2} \geq \ldots \geq W_{N}$, and introduce the momentum ratios

$$
\eta_{n}=\frac{W_{n}}{W_{n-1}} \quad(n=1, \ldots N) .
$$

Our product ansatz for the fragmentation function, which we will motivate in a moment, is:

$$
\begin{aligned}
D_{q}^{\pi}(z) & =\sum_{m=1}^{N} \int_{0}^{1} \mathrm{~d} \eta_{1} \int_{0}^{1} \mathrm{~d} \eta_{2} \ldots \int_{0}^{1} \mathrm{~d} \eta_{N} \sum_{Q_{N}} 6 d_{q}^{Q_{1}}\left(\eta_{1}\right) \cdot 6 d_{Q_{1}}^{Q_{2}}\left(\eta_{2}\right) \cdot \ldots \cdot 6 d_{Q_{N-1}}^{Q_{N}}\left(\eta_{N}\right) \\
& \times \delta\left(z-z_{m}\right) \delta\left(\tau_{\pi},\left(\tau_{Q_{m-1}}-\tau_{Q_{m}}\right) / 2\right) .
\end{aligned}
$$

Here the functions $d_{Q}^{Q^{\prime}}(\eta)$ are our elementary $Q \rightarrow Q^{\prime}$ splitting functions of 
Eq.(3.10), which represent the probability that a quark of flavor $Q$ makes a transition to the quark $Q^{\prime}$, leaving the momentum fraction $\eta$ to $Q^{\prime}$. A sum over repeated flavor indices in implied in (4.2); a flavor sum over the quark remainder $\left(Q_{N}\right)$ is included; for the case $N=1$ we define $Q_{0} \equiv q$; and the symbol $\delta(i, j)$ denotes the Kronecker delta. The factor 6 which multiplies each elementary splitting function comes from the sum over spin and color. The delta function in (4.2) selects a meson, which is produced in the m-th step with momentum fraction $z_{m}$ of the initial quark:

$$
\begin{aligned}
z_{m} & =\frac{W_{m-1}-W_{m}}{W_{0}}=\eta_{1} \cdot \eta_{2} \cdot \ldots \cdot \eta_{m-1} \cdot\left(1-\eta_{m}\right) \quad(m>1) \\
z_{1} & =1-\eta_{1}
\end{aligned}
$$

Because the pion has a mass, we will exclude the unphysical case of $z=0$, i.e., whenever a pion is produced in the $m$-th step we will assume that $\eta_{m} \neq 1$ in (4.3).

We will write the $q \rightarrow Q$ splitting function Eq.(3.10) including the spincolor factor 6 in the form

$$
\begin{aligned}
6 d_{q}^{Q}(z) & =Z_{Q} \delta(z-1) \delta_{q, Q}+F_{q}^{Q}(z) \\
F_{q}^{Q}(z) & =\left(\frac{1}{2}-\frac{\tau_{q} \tau_{Q}}{6}\right) F(z) \\
F(z) & =\frac{3}{2} g_{\pi}^{2}(1-z) \int \frac{\mathrm{d}^{2} p_{\perp}}{(2 \pi)^{3}} \frac{\boldsymbol{p}_{\perp}^{2}+M^{2}(1-z)^{2}}{\left[\boldsymbol{p}_{\perp}^{2}+M^{2}(1-z)^{2}+z M_{\pi}^{2}\right]^{2}}
\end{aligned}
$$

The function $F$ satisfies the normalization (see Eq.(3.11))

$$
\sum_{Q} \int_{0}^{1} \mathrm{~d} z F_{q}^{Q}(z)=\int_{0}^{1} \mathrm{~d} z F(z)=1-Z_{Q} .
$$

For the case $N=1$ it is easy to see that the function (4.2) reduces to the elementary fragmentation function (3.4) of the last section:

$$
\left.D_{q}^{\pi}(z) \stackrel{N=1}{\longrightarrow} F_{q}^{Q}(1-z)\right|_{\tau_{Q}=\tau_{q}-2 \tau_{\pi}}=\frac{1}{3}\left(1+\tau_{q} \tau_{\pi}\right) F(1-z)=d_{q}^{\pi}(z) .
$$


In order to show the physical content of the ansatz (4.2), we rewrite it identically as follows: Noting that each factor in the product (4.2) consists of the two terms in (4.4), it is easy to see that all products with the same number (call it $k$ ) of $F^{\prime} s$ and $(N-k)$ number of $Z_{Q}$ 's make the same contribution to $D_{q}^{\pi}(z)$. Therefore we can introduce an ordering of the $\eta$ 's in Eq.(4.2): Take the first $k \eta$ 's to be unequal $1\left(\eta_{1}, \eta_{2}, \ldots \eta_{k} \neq 1\right)$, and the remaining $\eta$ 's to be $1\left(\eta_{k+1}, \eta_{k+2}, \ldots \eta_{N}=1\right)$, multiply the combinatoric factor $\left(\begin{array}{c}N \\ k\end{array}\right)$, and perform a sum over $k$. For some fixed $k$, only terms with $m \leq k$ will contribute in the sum of Eq.(4.2), because $z_{m}$ of (4.3) must be non-zero. (As explained above, we consider only the case $z>0$.) Then Eq.(4.2) is rewritten identically as (see Fig.4)

$$
\begin{aligned}
D_{q}^{\pi}(z) & =\sum_{m=1}^{N} \sum_{k=m}^{N} P(k) \int_{0}^{1} \mathrm{~d} \eta_{1} \int_{0}^{1} \mathrm{~d} \eta_{2} \ldots \int_{0}^{1} \mathrm{~d} \eta_{k} \sum_{Q_{k}} \hat{F}_{q}^{Q_{1}}\left(\eta_{1}\right) \hat{F}_{Q_{1}}^{Q_{2}}\left(\eta_{2}\right) \ldots \hat{F}_{Q_{k-1}}^{Q_{k}}\left(\eta_{k}\right) \\
& \times \delta\left(z-z_{m}\right) \delta\left(\tau_{\pi},\left(\tau_{Q_{m-1}}-\tau_{Q_{m}}\right) / 2\right) \equiv \sum_{m=1}^{N} D_{q,(m)}^{\pi}(z)
\end{aligned}
$$

Here

$$
P(k)=\left(\begin{array}{c}
N \\
k
\end{array}\right) Z_{Q}^{N-k}\left(1-Z_{Q}\right)^{k}
$$

is the probability of producing $k$ mesons out of a maximum of $N$ mesons, and satisfies the normalization condition

$$
\sum_{k=0}^{N} P(k)=1 .
$$

In Eq.(4.9) we defined the renormalized function $\hat{F}_{q}^{Q} \equiv F_{q}^{Q} /\left(1-Z_{Q}\right)$, i.e., $($ see $(4.5)-(4.7))$

$$
\begin{aligned}
\hat{F}_{q}^{Q}(z) & =\left(\frac{1}{2}-\frac{\tau_{q} \tau_{Q}}{6}\right) \hat{F}(z), \quad \hat{F}(z)=\frac{F(z)}{1-Z_{Q}}, \\
\int_{0}^{1} \mathrm{~d} z \sum_{Q} \hat{F}_{q}^{Q}(z) & =\int_{0}^{1} \mathrm{~d} z \hat{F}(z)=1 .
\end{aligned}
$$




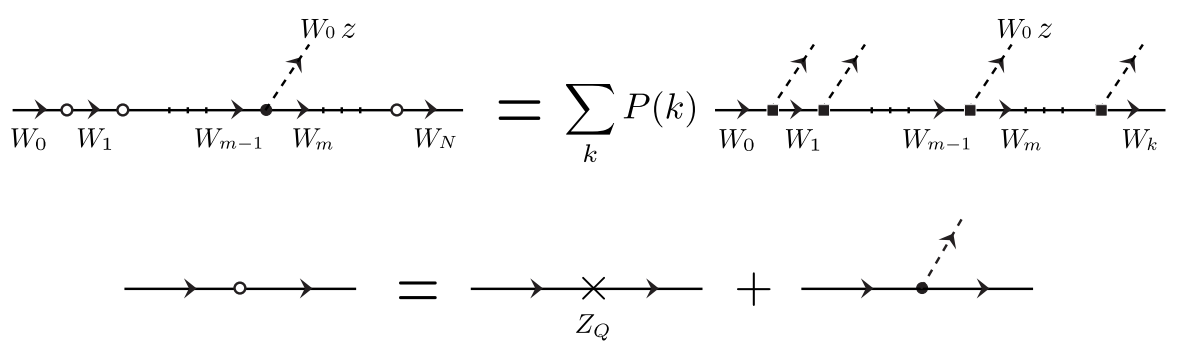

Figure 4: Graphical representation of the identity of Eq.(4.2) (1.h.s.) and Eq.(4.9) (r.h.s.). On the l.h.s., the open circles represent the elementary $q \rightarrow Q$ fragmentation function of Eq.(4.4), and the dots represent the second (meson emission) term in (4.4). In the $m$-th step, where a meson with momentum $W_{0} z$ is selected by the delta-function in (4.9), only the meson emission term contributes. On the r.h.s., $P(k)$ is the binomial distribution (4.10), and the squares represent the renormalized meson emission term given by Eq.(4.12).

The physical interpretation of (4.9) is as follows:

- $P(k)$ is the probability that $k$ mesons out of a maximum of $N$ mesons are produced.

- $\hat{F}_{Q}^{Q^{\prime}}(\eta)$ is the probability density that, if a meson is emitted from the quark $Q$, the momentum fraction $\eta$ is left to the remaining quark $Q^{\prime}$.

- The product $\hat{F}\left(\eta_{1}\right) \cdot \hat{F}\left(\eta_{2}\right) \cdot \ldots \hat{F}\left(\eta_{k}\right)$ is the probability density that, if $k$ mesons are produced, each meson carries its momentum fraction $z_{m}$ $(m=1, \ldots k)$ of the original quark, where $z_{m}$ is given by Eq.(4.3).

- $D_{q,(m)}^{\pi}(z)$ is the probability density that the $m$-th meson has the momentum fraction $z$ of the original quark. This implies that at least $m$ mesons must be produced, which corresponds to the lower limit $(k=m)$ in the summation in (4.9). The total fragmentation function $D_{q}^{\pi}(z)$ is then obtained by summing up the probability densities $D_{q,(m)}^{\pi}(z)$. 
We note that the original ansatz of Field and Feynman [17] is an infinite product, which formally emerges from Eq.(4.9) if we assume that $P(k)$ is equal to 1 for large $k \rightarrow \infty$, i.e., that an infinite number of mesons is emitted from the fragmenting quark.

Let us now continue with Eq.(4.9) to find the integral equation satisfied by the fragmentation function. For a fixed $m$, we can integrate over $\eta_{m+1}, \ldots \eta_{N}$ by using the normalization of $\hat{F}$, i.e.;

$$
\int_{0}^{1} \mathrm{~d} \eta \sum_{Q} \hat{F}_{q}^{Q}(\eta)=\int_{0}^{1} \mathrm{~d} \eta \int_{0}^{1} \mathrm{~d} \eta^{\prime} \sum_{Q^{\prime}} \hat{F}_{q}^{Q}(\eta) \hat{F}_{Q}^{Q^{\prime}}\left(\eta^{\prime}\right)=\ldots=1
$$

Then for all $k \geq m$ the integrations over the same variables $\eta_{1}, \ldots \eta_{m}$ remain, and the sum over $k$ refers only to the probabilities $P(k)$. Performing the shift $\eta_{m} \rightarrow 1-\eta_{m}$ in the integral over $\eta_{m}$, we obtain

$$
\begin{aligned}
D_{q(m)}^{\pi}(z) & =\left(\sum_{k=m}^{N} P(k)\right) \int_{0}^{1} \mathrm{~d} \eta_{1} \int_{0}^{1} \mathrm{~d} \eta_{2} \ldots \int_{0}^{1} \mathrm{~d} \eta_{m} \hat{F}_{q}^{Q_{1}}\left(\eta_{1}\right) \hat{F}_{Q_{1}}^{Q_{2}}\left(\eta_{2}\right) \ldots \hat{F}_{Q_{m-2}}^{Q_{m-1}}\left(\eta_{m-1}\right) \\
& \times \hat{d}_{Q_{m-1}}^{\pi}\left(\eta_{m}\right) \delta\left(z-\eta_{1} \eta_{2} \ldots \eta_{m}\right) .
\end{aligned}
$$

Here the function $\hat{d}_{q}^{\pi}(z) \equiv d_{q}^{\pi}(z) /\left(1-Z_{Q}\right)$ is the renormalized elementary $q \rightarrow \pi$ fragmentation function, i.e; (see (4.8))

$$
\hat{d}_{q}^{\pi}(z)=\left.\hat{F}_{q}^{Q}(1-z)\right|_{\tau_{Q}=\tau_{q}-2 \tau_{\pi}}=\frac{1}{3}\left(1+\tau_{q} \tau_{\pi}\right) \hat{F}(1-z) .
$$

From Eq.(4.15), it is easy to derive the following recursion relation for $m>1$ :

$$
D_{q(m)}^{\pi}(z)=R_{m}\left[\hat{F}_{q}^{Q} \otimes D_{Q,(m-1)}^{\pi}\right](z) \quad(m>1),
$$

while for $m=1$ we have

$$
D_{q(1)}^{\pi}(z)=R_{1} \hat{d}_{q}^{\pi}(z) .
$$

Here we introduced the following ratios:

$$
R_{n}=\frac{\sum_{k=n}^{N} P(k)}{\sum_{k=n-1}^{N} P(k)} \quad(n=1,2, \ldots N),
$$


and the following notation for the convolution of 2 functions $A(z)$ and $B(z)$ :

$$
[A \otimes B](z)=\int_{0}^{1} \mathrm{~d} z_{1} \int_{0}^{1} \mathrm{~d} z_{2} \delta\left(z-z_{1} z_{1}\right) A\left(z_{1}\right) B\left(z_{2}\right) .
$$

The total fragmentation function then becomes

$$
D_{q}^{\pi}(z)=R_{1} \hat{d}_{q}^{\pi}(z)+\sum_{n=2}^{N} R_{n}\left[\hat{F}_{q}^{Q} \otimes D_{Q(n-1)}^{\pi}\right](z),
$$

where $D_{q(m)}^{\pi}$ can be obtained from the recursion relation (4.17) with the starting value (4.18).

It is interesting at this stage to derive the sum rules for the fragmentation function. A simple calculation using Eq.(4.15) gives the following expression for the multiplicity, the momentum sum and the isospin sum:

$$
\begin{aligned}
\int_{0}^{1} \mathrm{~d} z \sum_{\tau_{\pi}} F_{q}^{\pi}(z) & =\sum_{k=1}^{N} k P(k)=N\left(1-Z_{Q}\right), \\
\int_{0}^{1} z \mathrm{~d} z \sum_{\tau_{\pi}} F_{q}^{\pi}(z) & =1-\sum_{k=0}^{N} P(k)\langle z \hat{F}\rangle^{k} \\
& =1-\left(Z_{Q}+\left(1-Z_{Q}\right)\langle z \hat{F}\rangle\right)^{N}, \\
\int_{0}^{1} \mathrm{~d} z \sum_{\tau_{\pi}} \tau_{\pi} F_{q}^{\pi}(z) & =\frac{\tau_{q}}{2}\left[1-\sum_{k=0}^{N} P(k)\left(-\frac{1}{3}\right)^{k}\right] \\
& =\frac{\tau_{q}}{2}\left[1-\left(Z_{Q}-\frac{1}{3}\left(1-Z_{Q}\right)\right)^{N}\right],
\end{aligned}
$$

where $\langle A\rangle \equiv \int_{0}^{1} \mathrm{~d} z A(z)$. These expressions can be understood as follows: If $k$ mesons are produced with probability $P(k)$, then Eq.(4.22) is simply the mean number of mesons; the quantity $P(k)\langle z \hat{F}\rangle^{k}$ in (4.23) is the mean momentum fraction left to the quark remainder; and the quantity $P(k)(-1 / 3)^{k}$ in (4.24) is the mean isospin fraction left to the quark remainder.

Eqs.(4.22) - (4.23) indicate that in the present model it is not possible to transfer the total momentum and isospin of the original quark to the 
mesons, if the maximum number of mesons is finite. The momentum and isospin sum rules (2.9) and (2.10) are valid only in the limit $N \rightarrow \infty$. While this may indicate a conceptual limitation of the jet model, we note that in general also the QCD based empirical analysis of fragmentation functions leads to divergent multiplicities. Therefore we find it more important to satisfy the momentum and isospin sum rules (2.9) and (2.10) than to have finite multiplicities, and take the limit $N \rightarrow \infty$. The results then become independent of the form of the distribution $P(k)$, if the following condition is satisfied for the ratios (4.19):

$$
R_{n} \stackrel{N \rightarrow \infty}{\longrightarrow} 1 \quad(\text { for all } n=1,2, \ldots)
$$

In fact, it is well known that in the limit $N \rightarrow \infty$ our binomial distribution (4.10) becomes a normalized Gauss distribution (normal distribution) $\frac{1}{\sqrt{2 \pi c^{2}}} e^{-\frac{\left(k-k_{0}\right)^{2}}{2 c^{2}}}$, with the same mean value $k_{0}=N\left(1-Z_{Q}\right)$ and variance $c^{2}=N Z_{Q}\left(1-Z_{Q}\right)$ as the original binomial distribution. The validity of (4.25) can then be easily confirmed. In fact, any distribution which approaches a normal distribution in the limit $N \rightarrow \infty$ satisfies the condition $(4.25)^{9}$.

Using (4.25), we see from (4.21) that our fragmentation function satisfies the same integral equation as in the original quark jet model [17]:

$$
D_{q}^{\pi}(z)=\hat{d}_{q}^{\pi}(z)+\left[\hat{F}_{q}^{Q} \otimes D_{Q}^{\pi}\right](z),
$$

where the driving term is given by (4.16), and the integral kernel by (4.12).

\footnotetext{
${ }^{9}$ The fact that in the limit $N \rightarrow \infty$ the binomial distribution becomes a normal distribution is known as the Moivre-Laplace theorem, which can be formulated rigorously in integral form ("weak convergence"). The central limit theorem [28] is an extension of the Moivre-Laplace theorem to general distributions $P(k)$ with mean value $\propto N$ and variance $c^{2} \propto N$. This indicates that Eq.(4.25) is actually valid for a wide class of distributions. Although our NJL-jet model ansatz (4.2) leads to the binomial distribution, in the limit $N \rightarrow \infty$ the results hold for a wide class of distributions.
} 
We finally write down the equations which will be solved in the next section: Defining two functions $A(z)$ and $B(z)$ by the isospin decomposition

$$
D_{q}^{\pi}(z) \equiv \frac{1}{3}\left(A(z)+\tau_{q} \tau_{\pi} B(z)\right),
$$

and using (4.12) and (4.16), we find the following integral equations for $A(z)$ and $B(z)$ from (4.26):

$$
\begin{aligned}
& A(z)=\hat{F}(1-z)+\int_{z}^{1} \frac{\mathrm{d} y}{y} \hat{F}\left(\frac{z}{y}\right) A(y), \\
& B(z)=\hat{F}(1-z)-\frac{1}{3} \int_{z}^{1} \frac{\mathrm{d} y}{y} \hat{F}\left(\frac{z}{y}\right) B(y),
\end{aligned}
$$

where $\hat{F}(z)$ is obtained by renormalizing the function $F(z)$ in (4.6) to 1 . Using (4.27), we obtain the following expressions for the "favored", "unfavored", and "neutral" fragmentation functions:

$$
\begin{aligned}
& D_{u}^{\pi^{+}}=D_{d}^{\pi^{-}}=D_{\bar{u}}^{\pi^{-}}=D_{\frac{\pi^{+}}{d}}=\frac{1}{3}(A+B), \\
& D_{u}^{\pi^{-}}=D_{d}^{\pi^{+}}=D_{\bar{u}}^{\pi^{+}}=D_{\frac{\pi^{-}}{d}}=\frac{1}{3}(A-B), \\
& D_{u}^{\pi^{0}}=D_{d}^{\pi^{0}}=D_{\frac{\pi^{0}}{u}}=D{\frac{\pi^{0}}{d}}^{0}=\frac{1}{3} A .
\end{aligned}
$$

From the form of the integral equations (4.28) and (4.29) it is easily seen that $\langle A\rangle=1,\langle B\rangle=3 / 4$, which leads to the momentum and isospin sum rules (2.9) and (2.10). For large $z$, both functions $A(z)$ and $B(z)$ go the $\hat{F}(1-z)$, and therefore the unfavored fragmentation functions (4.31) are suppressed for large pion momenta.

\section{Numerical results and discussions}

In this section we present the numerical results for the fragmentation function of Eq.(4.26) in the NJL-jet model. For reference, we also give the well known results for the elementary distribution function of Eq.(3.2). 
Because the use of the NJL model to calculate the quark distributions in the pion has been explained in detail in Ref.[24], we will not repeat the explanations of the model here. For convenience, we will use the same regularization scheme, namely the invariant mass, or Lepage-Brodsky (LB) [29], regularization scheme with the same parameters as in Ref.[24]. The LB scheme is suitable for regularizing integrals in terms of light-cone variables, and in terms of usual variables it is equivalent to the familiar 3-momentum cut-off scheme [24]. Namely, if we denote the 3-momentum cut-off by $\Lambda_{3}$, which is fixed in the usual way by reproducing the experimental pion decay constant, a bubble-type loop integral with two intermediate particles of mass $M_{1}$ and $M_{2}$ is regularized by cutting off their invariant mass $M_{12}$ according to

$$
M_{12} \leq \Lambda_{12} \equiv \sqrt{\Lambda_{3}^{2}+M_{1}^{2}}+\sqrt{\Lambda_{3}^{2}+M_{1}^{2}}
$$

In terms of light cone variables, if we associate to particle 1 the fraction $y$ of the total $P_{-}$and transverse momentum $\boldsymbol{q}_{T}$, and to particle 2 the fraction $(1-y)$ and $-\boldsymbol{q}_{T}$, their invariant mass squared is

$$
M_{12}^{2}=\frac{M_{1}^{2}+\boldsymbol{q}_{T}^{2}}{y}+\frac{M_{2}^{2}+\boldsymbol{q}_{T}^{2}}{1-y}
$$

The requirement $M_{12} \leq \Lambda_{12}$ then leads to a $y$-dependent transverse cut-off: $\boldsymbol{q}_{T}^{2} \leq \Lambda_{12}^{2} y(1-y)-M_{1}^{2}(1-y)-M_{2}^{2} y$. This condition also restricts the values of $y$ from below and above $\left(0<y_{1} \leq y \leq y_{2}<1\right)$. For example, for the integral (3.4) of the elementary $q \rightarrow \pi$ fragmentation function we have $M_{1}=M_{\pi}, M_{2}=M$, and for the integral (3.10) of the elementary $q \rightarrow Q$ fragmentation function we have $M_{1}=M, M_{2}=M_{\pi}$. For the integral (3.2) of the distribution function we have $M_{1}=M_{2}=M$. We also note that this regularization schemes does not violate the sum rules. 
As in Ref.[24], we will use the constituent quark mass $M=300 \mathrm{MeV}$. Then $\Lambda_{3}=670 \mathrm{MeV}$, and the invariant mass cut-offs for the $(\pi, q)$ and $(q, q)$ systems are $1.42 \mathrm{GeV}$ and $1.47 \mathrm{GeV}$, respectively. We did not investigate whether other parameter sets or other regularization schemes lead to a better description of the fragmentation functions.

As usual, we will associate a low energy renormalization scale $\left(Q_{0}^{2}\right)$ to our NJL results, and evolve them in $Q^{2}$ by using the evolution equations. For the evolution of the fragmentation functions, we limit ourselves to the LO. In this case, it has been verified [15] that a formal application of the DLY relation (2.11) leads to the correct connection between the evolution kernels for the distribution and fragmentation functions (see Appendix B), although the DLY relation is actually not used to relate the distribution and fragmentation functions themselves. We therefore use the $Q^{2}$ evolution code of Ref.[30] in LO for the distribution functions, and perform the transformation of the kernels as explained in Appendix B to obtain the LO evolution of the fragmentation functions ${ }^{10}$.

First, in Fig. 5a we recapitulate the results of Fig. 4 of Ref.[24] for the valence $u$ quark distribution ("minus-type" $u-\bar{u}$ ) in $\pi^{+}$, and in Fig. 5b we show the "plus-type" distribution $(u+\bar{u})$. The dashed line shows the NJL model result based on Eq.(3.2), the solid lines shows the distribution obtained by associating a low energy scale of $Q_{0}^{2}=0.18 \mathrm{GeV}^{2}$ to the NJL result and performing the $Q^{2}$ evolution in LO and NLO to $Q^{2}=4 \mathrm{GeV}^{2}$, and the dotted line shows the empirical NLO parametrizations of Ref.[8]. We

\footnotetext{
${ }^{10}$ The DLY based relation between the evolution kernels for distribution and fragmentation functions is violated in NLO [15]. Unfortunately, a NLO evolution code for the fragmentation functions is not yet available to the public. Because in this paper we do not attempt a quantitative comparison with the empirical functions, we leave the NLO calculation for a future work.
} 
see that the LO and NLO results show quantitative differences because of the rather low value assumed for $Q_{0}^{2}$, although the qualitative behaviors are similar.
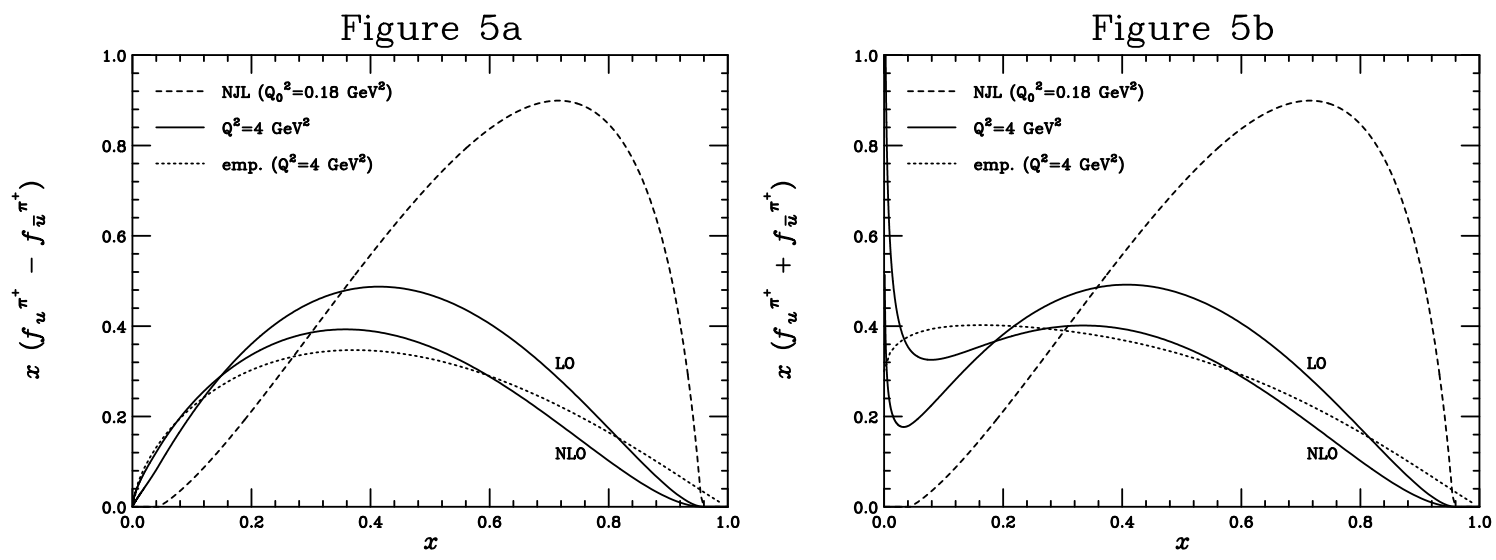

Figure 5: (a) "Minus-type" (valence) distribution $x\left(f_{u}^{\pi^{+}}(x)-f \frac{\pi^{+}}{u}(x)\right)$, and (b) "plus-type" distribution $x\left(f_{u}^{\pi^{+}}(x)+f_{\frac{\pi}{u}}^{+}(x)\right)$ of $u$ quark in $\pi^{+}$. The dashed line is the NJL model result, used as input $\left(Q_{0}^{2}=0.18 \mathrm{GeV}^{2}\right)$ for the $Q^{2}$ evolution. The solid line labeled by LO (NLO) is the result of the evolution to $Q^{2}=4 \mathrm{GeV}^{2}$ in LO (NLO). The dotted line is the empirical NLO result of Ref.[8] at $Q^{2}=4 \mathrm{GeV}^{2}$.

In Fig. 6 we show the corresponding results for the minus-type and plustype fragmentation functions for $u \rightarrow \pi^{+}$. Here the NJL-jet result shown by the dashed line is the solution of the integral equation (4.26), i.e., the dashed line in Fig.6a (Fig. 6b) shows the function $\frac{2}{3} B(z)\left(\frac{2}{3} A(z)\right)$, see Eqs.(4.30) and (4.31). In order to see the importance of the cascade processes, we also show the driving term of the integral equation (the function $\frac{2}{3} \hat{F}(1-z)$ ) by the upper dash-double-dotted line, which is the renormalized elementary fragmentation function $\left(\frac{2}{3} F(1-z)\right)$ shown by the lower dash-double-dotted line. The result of the evolution of the dashed line $\left(Q_{0}^{2}=0.18 \mathrm{GeV}^{2}\right)$ to $Q^{2}=4$ $\mathrm{GeV}^{2}$ in LO is shown by the solid line, and the dotted line shows the empiri- 
cal NLO result of Ref.[10], evolved to $Q^{2}=4 \mathrm{GeV}^{2}$. Several important points
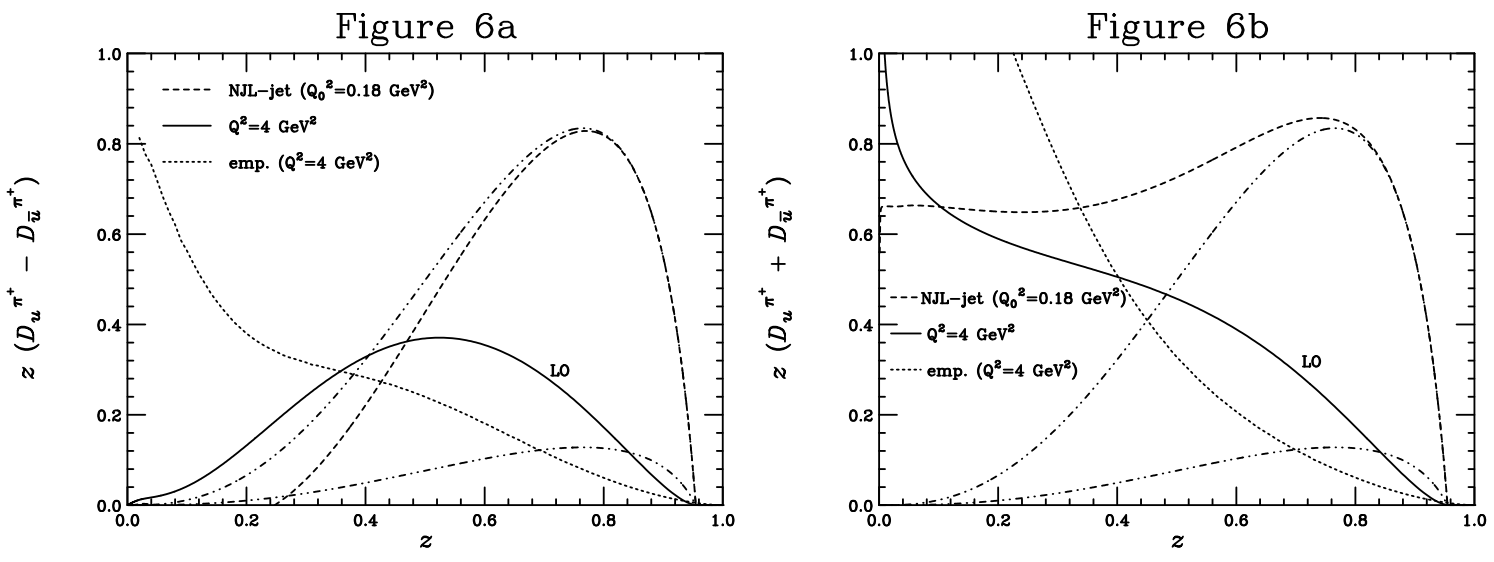

Figure 6: (a) "Minus-type" $z\left(D_{u}^{\pi^{+}}(z)-D_{\frac{\pi^{+}}{u}}(z)\right)$, and (b) "plus-type" $z\left(D_{u}^{\pi^{+}}(z)+D_{\bar{u}}^{\pi^{+}}(z)\right)$ fragmentation functions for $u \rightarrow \pi^{+}$. The dashed line is the NJL-jet model result, used as input $\left(Q_{0}^{2}=0.18 \mathrm{GeV}^{2}\right)$ for the $Q^{2}$ evolution. The lower dash-double-dotted line is the elementary fragmentation function ( $d$ of Eq.(3.4)), and the upper dash-double-dotted line is the renormalized elementary fragmentation function ( $\hat{d}$ of Eq.(4.16)), which is the driving term of the integral equation (4.26). The solid line labeled as LO is the result of the evolution to $Q^{2}=4 \mathrm{GeV}^{2}$ in LO. The dotted line is the empirical NLO result of Ref.[10], evolved to $Q^{2}=4 \mathrm{GeV}^{2}$.

can be seen from Fig.6: First, as was anticipated in Sect.3, the elementary fragmentation function (lower dash-double-dotted line) is very small. Second, Fig. 6b shows the tremendous enhancement at intermediate and small $z$ of the plus-type fragmentation function caused by the cascade processes (iterations of integral equation (4.26)), while for the minus-type fragmentation function of Fig.6a a small reduction is seen. Third, the calculated result shown by the solid line has the correct order of magnitude for intermediate and large $z$, compared to the empirical function. This point, which reflects the fact that our model satisfies the momentum sum rule, is very important, because effective quark model calculations done so far considered only the 
elementary fragmentation functions, and introduced some ad-hoc parameters (like normalization constants) in order to get the correct order of magnitude. Quantitatively, Figure 6 indicates that our fragmentation functions are too large at large $z$ and too small at smaller $z$. This is natural, because we can expect that a NLO calculation will lead to a softening of the fragmentation functions, and also the coupling to other fragmentation channels (in particular the nucleon, antinucleon and kaon) will transfer some amount of the hard quark momentum to the other hadrons. Also, one should not forget that the empirical fragmentation functions have very large uncertainties, which are not indicated in our figures. Nevertheless, Figure 6 indicates that the present NJL-jet model provides a reasonable starting point for the description of fragmentation functions.
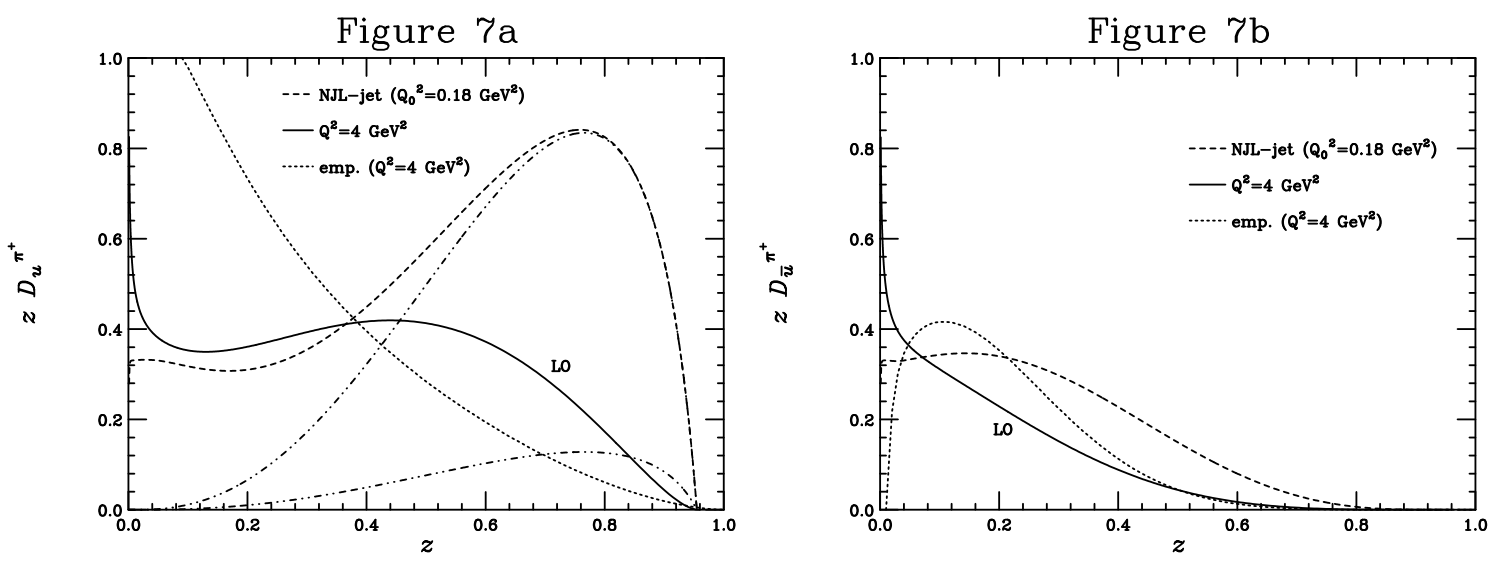

Figure 7: (a) "Favored" $z D_{u}^{\pi^{+}}(z)$, and (b) "unfavored" $z D_{\bar{u}}^{\pi^{+}}(z)$ fragmentation functions. In Fig. 7(a), the lower dash-double-dotted line is the elementary fragmentation function ( $d$ of Eq.(3.4)), and the upper dashdouble-dotted line is the renormalized elementary fragmentation function ( $\hat{d}$ of Eq.(4.16)), which is the driving term of the integral equation (4.26). (These functions are zero for the unfavored case.) The solid line labeled as LO is the result after evolution to $Q^{2}=4 \mathrm{GeV}^{2}$ in LO. The dotted line is the empirical NLO result of Ref.[10], evolved to $Q^{2}=4 \mathrm{GeV}^{2}$. 
Fig. 7a shows the results for the favored fragmentation function of Eq.(4.30), and Fig.6b shows the unfavored fragmentation function of Eq.(4.31). (These figures correspond to half of the sum and half of the difference of the curves in Fig.6a and 6b.) The upper dash-double-dotted line in Fig.7a shows the driving term $\left(\frac{2}{3} \hat{F}(1-z)\right)$ of the integral equation (4.26), and the lower dash-double-dotted line shows the elementary fragmentation function $\left(\frac{2}{3} F(1-z)\right)$. (For the unfavored case, these two functions are zero.) Both figures demonstrate the importance of cascade processes in the present NJL-jet model.

\section{Summary and conclusions}

In this paper we used the NJL model as an effective quark theory to study the simplest fragmentation function, i.e., the fragmentation of unpolarized quarks to pions. Our aim was to provide a framework which satisfies the momentum and isospin sum rules in a natural way without introducing adhoc parameters, and which gives the correct order of magnitude compared to the empirical fragmentation functions at intermediate and large $z$. We explained in detail that, for this purpose, the simplest approximation, namely the truncation to the one-quark spectator state in the defining relation (2.2), is completely inadequate: Although this approximation does not violate any conservation laws, it gives only very small fragmentation functions, because the probability for the elementary fragmentation process is small in effective theories based on constituent quarks, and the quark remainder can carry an appreciable amount of momentum.

In order to overcome these difficulties, we followed the idea of the quark jet model, and made a product ansatz to describe the cascade processes in the 
NJL model. We explained that this ansatz corresponds to the assumption of a binomial distribution for the number of mesons emitted from the quark, but in the limit that the maximum number of mesons becomes very large the results are independent of the assumed distribution function. Our formulation thus represents an extension of the original quark jet model, which assumes an infinite number of mesons from the outset. We have shown in detail that this NJL-jet model describes fragmentation processes where $100 \%$ of the initial quark light cone momentum is transferred to mesons. The momentum sum rule (2.9), which is supposed to be valid in all QCD based empirical fits, is then satisfied naturally without introducing any new parameters into the theory. We have also shown that the isospin sum rule (2.10) is naturally satisfied in this approach.

The comparison with the empirical fragmentation functions shows that our calculated functions have the correct order of magnitude for intermediate and large $z$. We pointed out that a straight forward extension to include the NLO terms in the $Q^{2}$ evolution, and to include other fragmentation channels besides the pion, will improve the description. Therefore we can conclude that our NJL-jet model provides a reasonable framework to analyse fragmentation functions in an effective quark theory.

For future works in this direction, it is important to derive the jet model type product ansatz from field theory. One can expect that the rainbowladder scheme for the quark self energy provides a suitable framework for this purpose. One may attempt to use this truncation scheme consistently to describe the cascade processes for the fragmentation functions, and the contributions of the hadron cloud around the quark for the distribution functions. One has to bear in mind, however, that a truncation scheme which 
works well for fragmentation processes may not be suitable for the distribution functions, and vice versa. To establish a scheme which respects the sum rules and which gives a satisfactory description of both type of processes is an important task for future research.

\section{ACKNOWLEDGEMENTS}

The authors thank A. Bacchetta, H. Hirai, T.-H. Nagai, M. Stratmann, and K. Tanaka for helpful discussions. W.B. and T.I. acknowledge the hospitality of Jefferson National Laboratory and Argonne National Laboratory, where part of this work has been done during spring and summer 2008. This work was supported by the Grant in Aid for Scientific Research of the Japanese Ministry of Education, Culture, Sports, Science and Technology, Project No. C-19540306, by the U.S. Department of Energy Grant No. DEFG0397ER4014, and by the Contract No. DE-AC05-06OR23177, under which Jefferson Science Associates, LLC operates Jefferson Laboratory. 


\section{References}

[1] R.D. Field, R.P. Feynman, Phys. Rev. D 15 (1977) 2590;

F.E. Close, An Introduction to Quarks and Partons, Academic Press, 1979.

[2] G. Altarelli, R.K. Ellis, M. Martinelli, S.-Y. Pi, Nucl. Phys. B 160 (1979) 301.

[3] J.C. Collins, D.E. Soper, Nucl. Phys. B 194 (1982) 445.

[4] R.L. Jaffe, Spin, Twist and Hadron Structure in Deep Inelastic Processes, International School of Nucleon Structure, Erice, 1995; hep$\mathrm{ph} / 9602236$.

[5] R.K. Ellis, W.J. Stirling, B.R. Webber, QCD and Collider Physics, Cambridge Univ. Press, 1996.

[6] V. Barone, A. Drago, P.G. Ratcliffe, Phys. Rept. 359 (2002) 1.

[7] A.D. Martin, R.G. Roberts, W.J. Stirling, R.S. Thorne, Eur. Phys. J. C 35 (2004) 325.

M. Hirai, S. Kumano, N. Saito, Phys. Rev. D 69 (2004) 054021.

[8] P.J. Sutton, A.D. Martin, R.G. Roberts, W.J. Stirling, Phys. Rev. D 45 (1992) 2349.

[9] I.C. Cloet, W. Bentz, A.W. Thomas, Phys. Lett. B 621 (2005) 246;

M. Wakamatsu, T. Kubota, Phys. Rev. D 57 (1998) 5755;

M.B. Hecht, C.D. Roberts, S.M. Schmidt, Phys. Rev. C 63 (2001) 025213 . 
[10] M. Hirai, S. Kumano, T.-H. Nagai, K. Sudoh, Phys. Rev. D 75 (2007) 094009;

D. de Florian, R. Sassot, M. Stratmann, Phys. Rev. D 75 (2007) 114010;

S. Kretzer, E. Leader, E. Christova, Eur. Phys. J. C 22 (2001) 269.

[11] J. Rajston, D.E. Soper, Nucl. Phys. B 152 (1979) 109.

[12] D.W. Sivers, Phys. Rev. D 41 (1990) 83;

J.C. Collins, Nucl. Phys. B 396 (1993) 161;

D. Boer, P.J. Mulders, F. Pijlman, Nucl. Phys. B 667 (2003) 201.

[13] J.T. Londergan, A. Pang, A.W. Thomas, Phys. Rev. D 54 (1996) 3154 ;

R. Jakob, P.J. Mulders, J. Rodrigues, Nucl. Phys. A 626 (1997) 937;

H. Kitagawa, Y. Sakemi, Prog. Theoret. Phys. 104 (2000) 421;

J.-J. Yang, Phys. Rev. D 65 (2002) 094035;

D. Amrath, A. Bacchetta, A. Metz, Phys. Rev. D 71 (2005) 114018;

A. Bacchetta, L. P. Gamberg, G.R. Goldstein, A. Mukherjee, Phys. Lett. B 659 (2008) 234.

[14] S.D. Drell, D.J. Levy, T.-M. Yan, Phys. Rev. 187 (1969) 2159; D1 (1970) 1035; D1 (1970) 1617.

[15] J. Blumlein, V. Radvindran, W.L. van Neerven, Nucl. Phys. B 586 (2000) 349 .

[16] C. Boros, J.T. Londergan, A.W. Thomas, Phys. Lett. B 473 (2000) 305.

[17] R.D. Field, R.P. Feynman, Nucl. Phys. B 136 (1978) 1; see also: T.D. Gottschalk, Hadronization and Fragmentation, 19th Int. School of Elementary Particle Physics, Kupari-Dubrovnik, 1983. 
[18] B. Andersson, G. Gustafson, B. Söderberg, Nucl. Phys. B 264 (1986) 29.

[19] R.D. Field, S. Wolfram, Nucl. Phys. B 213 (1983) 65.

[20] Y. Nambu, G. Jona-Lasinio, Phys. Rev. 122 (1960) 345; 124 (1961) 246.

[21] J. Collins, Nucl. Phys. B 396 (1993) 161.

[22] R.L. Jaffe, X.-D. Ji, Nucl. Phys. 375 (1992) 527.

[23] M. Burkardt, Adv. Nucl. Phys. 23 (1996) 1.

[24] W. Bentz, T. Hama, T. Matsuki, K. Yazaki, Nucl. Phys. A 651 (1999) 143.

[25] G. Altarelli, G. Parisi, Nucl. Phys. B 126 (1977) 298.

[26] M. Stratmann, W. Vogelsang, Nucl. Phys. B 496 (1997) 41.

[27] R.L. Jaffe, Nucl. Phys. B 229 (1983) 205.

[28] P.J. Huber, Robust statistics, Wiley, New York, 1981.

[29] G.P. Lepage, S.J. Brodsky, Phys. Rev. D 22 (1980) 2157.

[30] M. Miyama, S. Kumano, Comp. Phys. Commun. 94 (1996) 185. 


\section{A Proof of the DLY relation}

In this Appendix, we will prove the DLY relation (2.11) in two independent ways: First we follow the original derivation [14] in terms of the hadronic tensors, and second we start from the operator definitions (2.1) and (2.2). The formulae in this Appendix refer to the case where $h$ is a proton, in order to indicate the spinor algebra, but it is trivial to modify the expressions for the case where $h$ is a pion.

\section{A.1 General crossing relations}

We consider the following Green function

$$
\bar{M}_{\beta}^{a}\left(p, p_{n}\right)=\int \mathrm{d}^{4} x e^{-i p \cdot x}<p_{n}\left|T\left(\mathcal{O}^{a}(0) \bar{\Phi}_{\beta}(x)\right)\right| 0>,
$$

where $\Phi_{\beta}(x)$ is an interpolating field for the nucleon, and $\mathcal{O}^{a}$ is another local field operator. We also define the N-amputated Green function by $\bar{M}_{\beta}^{a}\left(p, p_{n}\right)=\bar{\Gamma}_{\gamma}^{a}\left(p, p_{n}\right) i G_{N, \gamma \beta}(p)$, where $G_{N}$ is the nucleon propagator. From the spectral representation of (A.1), or from the familiar reduction formalism, we can derive the relations

$$
\begin{aligned}
<p_{n}\left|\mathcal{O}^{a}\right| p> & =\bar{\Gamma}^{a}\left(p, p_{n}\right) \sqrt{2 M_{N}} u_{N}(\boldsymbol{p} s) \\
<\bar{p}, p_{n}\left|\mathcal{O}^{a}\right| 0> & =( \pm) \bar{\Gamma}^{a}\left(-p, p_{n}\right) \sqrt{2 M_{N}} v_{N}(\boldsymbol{p} s) .
\end{aligned}
$$

In (A.3) the sign is $(+)$ if $\mathcal{O}$ is a fermion type operator, and $(-)$ if it is a boson type operator. Also, $\bar{p}$ denotes an antinucleon with 4-momentum $p^{\mu}=$ $\left(E_{N}(\boldsymbol{p}), \boldsymbol{p}\right)$. The nucleon spinors are denoted as $u_{N}$ and $v_{N}$. Our covariant normalization implies the following matrix elements of the nucleon field operator: $<0|\Phi(0)| p>=\sqrt{2 M_{N}} u_{N}(\boldsymbol{p} s), \quad<\bar{p}|\Phi(0)| 0>=\sqrt{2 M_{N}} v_{N}(\boldsymbol{p} s)$. Eqs. (A.2), (A.3) are the basic crossing relations which will be used in the following. 


\section{A.2 Comparison of hadronic tensors}

Here we use the above crossing relations to find the connection between the hadronic tensors (spin independent parts only) for the processes $e h \rightarrow e^{\prime} X$ and $e^{+} e^{-} \rightarrow h X$, where $h$ denotes a hadron (proton) [22]:

$$
\begin{aligned}
W_{h}^{\mu \nu}(p, q) & =\frac{1}{4 \pi} \hat{\sum}_{n}(2 \pi)^{4} \delta^{4}\left(q+p-p_{n}\right)<p\left|J^{\mu}\right| p_{n}><p_{n}\left|J^{\nu}\right| p> \\
& =\left(-g^{\mu \nu}+\frac{q^{\mu} q^{\nu}}{q^{2}}\right) F_{1}^{h}\left(x, q^{2}\right)+\frac{1}{p \cdot q}\left(p^{\mu}-\frac{p \cdot q}{q^{2}} q^{\mu}\right)\left(p^{\nu}-\frac{p \cdot q}{q^{2}} q^{\nu}\right) F_{2}^{h}\left(x, q^{2}\right),
\end{aligned}
$$

$$
\begin{aligned}
\bar{W}_{h}^{\mu \nu}(p, q) & =\frac{1}{4 \pi} \hat{\sum}_{n}(2 \pi)^{4} \delta^{4}\left(q-p-p_{n}\right)<0\left|J^{\mu}\right| p, \bar{p}_{n}><p, \bar{p}_{n}\left|J^{\nu}\right| 0> \\
& =\left(-g^{\mu \nu}+\frac{q^{\mu} q^{\nu}}{q^{2}}\right) \bar{F}_{1}^{h}\left(z, q^{2}\right)+\frac{1}{p \cdot q}\left(p^{\mu}-\frac{p \cdot q}{q^{2}} q^{\mu}\right)\left(p^{\nu}-\frac{p \cdot q}{q^{2}} q^{\nu}\right) \bar{F}_{2}^{h}\left(z, q^{2}\right) .
\end{aligned}
$$

Here $|p\rangle$ is the state of the hadron $h$ with momentum $p$, and we use $x=\frac{-q^{2}}{2 p \cdot q}$ and $z=\frac{2 p \cdot q}{q^{2}}=-\frac{1}{x}$. We also defined $\hat{\sum}_{n}=\sum_{n} \int \frac{\mathrm{d}^{4} p_{n}}{(2 \pi)^{3}} \delta\left(p_{n}^{2}-M_{n}^{2}\right) \Theta\left(p_{n 0}\right)$, where $M_{n}$ is the invariant mass of the state $n$.

We now use (A.2) and its c.c. for the current operator $J^{\nu}$ :

$$
\begin{aligned}
& <p_{n}\left|J^{\nu}\right| p>=\sqrt{2 M_{N}} \bar{\Gamma}^{\nu}\left(p, p_{n}\right) u_{N}(\boldsymbol{p} s) \\
& <p\left|J^{\nu}\right| p_{n}>=\sqrt{2 M_{N}} \bar{u}_{N}(\boldsymbol{p} s) \Gamma^{\nu}\left(p, p_{n}\right)
\end{aligned}
$$

where $\Gamma_{\beta}^{\nu}=\left(\gamma_{0} \bar{\Gamma}^{\dagger \nu}\right)_{\beta}$ (that is, $\left.\bar{\Gamma}^{\nu}=\Gamma^{\nu \dagger} \gamma^{0}\right)$. We insert these relations into (A.4). Since we consider the spin independent part only, we can sum over the nucleon spin $s$ and divide by 2 , using $\sum_{s} u_{N}(\boldsymbol{p} s) \bar{u}_{N}(\boldsymbol{p} s)=\frac{\not p+M_{N}}{2 M_{N}}$. This gives

$4 \pi W_{h}^{\mu \nu}(p, q)=\frac{1}{2} \hat{\sum}_{n}(2 \pi)^{4} \delta^{4}\left(q+p-p_{n}\right) \operatorname{Tr}\left[\left(\not p+M_{N}\right) \Gamma^{\mu}\left(p, p_{n}\right) \bar{\Gamma}^{\nu}\left(p, p_{n}\right)\right]$. 
For the hadronic tensor in (A.5), we first use charge conjugation and then (A.3) and its c.c. for the current operator $J^{\mu}$ :

$$
\begin{aligned}
<0\left|J^{\mu}\right| p, \overline{p_{n}}> & =<0\left|\mathcal{C}^{-1}\left(\mathcal{C J}^{\mu} \mathcal{C}^{-1}\right) \mathcal{C}\right| p, \overline{p_{n}}>=<0\left|\left(\mathcal{C J}^{\mu} \mathcal{C}^{-1}\right)\right| \bar{p}, p_{n}> \\
& =-<0\left|J^{\mu}\right| \bar{p}, p_{n}>=\sqrt{2 M_{N}} \bar{v}_{N}(\boldsymbol{p} s) \Gamma^{\mu}\left(-p, p_{n}\right) \\
<p, \overline{p_{n}}\left|J^{\mu}\right| 0> & =-<\bar{p}, p_{n}\left|J^{\mu}\right| 0>=\sqrt{2 M_{N}} \bar{\Gamma}^{\mu}\left(-p, p_{n}\right) v_{N}(\boldsymbol{p} s)
\end{aligned}
$$

We insert these relations into (A.5), sum over the nucleon spin $s$ and divide by 2 using $\sum_{s} v_{N}(\boldsymbol{p} s) \bar{v}_{N}(\boldsymbol{p} s)=-\frac{-\not p+M_{N}}{2 M_{N}}$. This gives $4 \pi \bar{W}_{h}^{\mu \nu}(p, q)=-\frac{1}{2} \hat{\sum}_{n}(2 \pi)^{4} \delta^{4}\left(q-p-p_{n}\right) \operatorname{Tr}\left[\left(-\not p+M_{N}\right) \Gamma^{\mu}\left(-p, p_{n}\right) \bar{\Gamma}^{\nu}\left(-p, p_{n}\right)\right]$.

By comparing (A.8) with (A.9) we obtain the DLY crossing relation for the hadronic tensors:

$$
\bar{W}_{h}^{\mu \nu}(p, q)=-W_{h}^{\mu \nu}(-p, q) . \quad\left(s_{h}=\frac{1}{2}\right)
$$

The minus sign in (A.10) comes from the Dirac algebra explained above, and for a spinless hadron the minus sign is changed to plus. Eq. (A.10) implies the following relation ${ }^{11}$ between the structure functions in (A.4) and (A.5):

$$
\begin{aligned}
& \bar{F}_{1}^{h}\left(z, q^{2}\right)=-F_{1}^{h}\left(-x, q^{2}\right)=-F_{1}^{h}\left(\frac{1}{z}, q^{2}\right), \\
& \bar{F}_{2}^{h}\left(z, q^{2}\right)=F_{2}^{h}\left(-x, q^{2}\right)=F_{2}^{h}\left(\frac{1}{z}, q^{2}\right) .
\end{aligned}
$$

The well known relation $F_{2}^{h}(x)=2 x F_{1}^{h}(x)$ becomes, with $x \rightarrow-x$ and using the first equalities in (A.11) and (A.12):

$$
\bar{F}_{2}^{h}(z)=-\frac{2}{z} \bar{F}_{1}^{h}(z)
$$

\footnotetext{
${ }^{11} \mathrm{By}$ relations like $(\mathrm{A} .11)$ we mean the following: Take a particular physical value of $z$ for the $\left(e^{+}, e^{-}\right)$process $(0<z<1)$. Then the corresponding (unphysical) value of the Bjorken variable for the $\left(e, e^{\prime}\right)$ process is $x=1 / z$, and (A.11) gives the connection between the structure functions.
} 
which holds also for spinless bosons.

The connection between the structure function $\bar{F}_{1}^{h}$ and the fragmentation function $D_{q}^{h}(z)$ in the Bjorken limit is as follows: The cross section for the process $e^{+} e^{-} \rightarrow h X$ is [4] ${ }^{12}$

$$
\frac{\mathrm{d} \sigma^{h}}{\mathrm{~d} z}=\frac{2 \alpha^{2} \pi z}{q^{2}}\left(\bar{F}_{1}^{h}\left(z, q^{2}\right)+\frac{z}{6} \bar{F}_{2}^{h}\left(z, q^{2}\right)\right)=\frac{4}{3} \frac{\alpha^{2} \pi z}{q^{2}} \bar{F}_{1}^{h}(z) .
$$

Usually this is divided by the total cross section for $e^{+} e^{-} \rightarrow$ hadrons

$$
\sigma_{\text {tot }}=\frac{4 \pi \alpha^{2}}{q^{2}} \sum_{q} e_{q}^{2} \equiv \frac{4 \pi \alpha^{2}}{3 q^{2}} R,
$$

where $\sum_{q}$ refers to the quark flavor only. Then we obtain

$$
\frac{1}{\sigma_{\text {tot }}} \frac{\mathrm{d} \sigma^{h}}{\mathrm{~d} z}=\frac{1}{R} z \bar{F}_{1}^{h}(z) .
$$

This is compared to the original definition of the fragmentation function [1]:

$$
\frac{1}{\sigma_{\text {tot }}} \frac{\mathrm{d} \sigma^{h}}{\mathrm{~d} z} \equiv \frac{1}{R} 3 \sum_{q} e_{q}^{2}\left(D_{q}^{h}(z)+D_{\bar{q}}^{h}(z)\right)
$$

to obtain

$$
\bar{F}_{1}^{h}(z)=\frac{3}{z} \sum_{q} e_{q}^{2}\left(D_{q}^{h}(z)+D_{\bar{q}}^{h}(z)\right) .
$$

Because we know how to express $F_{1}^{h}(x)$ in the Bjorken limit by the distribution functions $f_{q}^{h}(x)$, we get from (A.11):

$$
\bar{F}_{1}^{h}(z)=-F_{1}^{h}\left(\frac{1}{z}\right)=-\frac{1}{2} \sum_{q} e_{q}^{2}\left(f_{q}^{h}\left(\frac{1}{z}\right)+f_{\bar{q}}^{h}\left(\frac{1}{z}\right)\right) .
$$

\footnotetext{
${ }^{12}$ We remind that the symbol $h$ denotes a particular hadron with a specified spin direction, e.g., $p \uparrow$ (although the spin averaged cross section considered here does not depend on the spin direction). Therefore, the cross section measured for the case that the spin of the produced nucleon is not observed has an additional factor of 2 , which is not included in (A.14).
} 
Comparing (A.18) and (A.19) we get

$$
D_{q}^{h}(z)=-\frac{z}{6} f_{q}^{h}\left(\frac{1}{z}\right), \quad\left(s_{h}=\frac{1}{2}\right)
$$

and similar for the antiquarks, which expresses the DLY relation (2.11) between the distribution and fragmentation functions. (For the case of a spinless hadron, the minus sign in (A.20) is changed to plus.)

\section{A.3 Comparing the operator definitions}

We now start from the operator definitions (2.1) and (2.2), which give

$$
\begin{aligned}
f_{q}^{h}(x) & =\frac{1}{2} \hat{\sum}_{n} \delta\left(p_{-} x-p_{-}+p_{n-}\right)<p|\bar{\psi}| p_{n}>\gamma^{+}<p_{n}|\psi| p> \\
D_{q}^{h}(z) & =\frac{z}{6} \frac{1}{2} \hat{\sum}_{n} \delta\left(\frac{p_{-}}{z}-p_{-}-p_{n-}\right)<p, \overline{p_{n}}|\bar{\psi}| 0>\gamma^{+}<0|\psi| p, \overline{p_{n}}>
\end{aligned}
$$

For definiteness we consider again the case where $h$ is a proton. We use $\mathcal{O}^{a}=\psi_{\alpha}$ in (A.1), which gives

$$
\begin{aligned}
<p_{n}|\psi| p> & =\bar{\Gamma}\left(p, p_{n}\right) \sqrt{2 M_{N}} u_{N}(\boldsymbol{p} s) \\
<\bar{p}, p_{n}|\psi| 0> & =\bar{\Gamma}\left(-p, p_{n}\right) \sqrt{2 M_{N}} v_{N}(\boldsymbol{p} s) .
\end{aligned}
$$

We insert (A.23) and its c.c. into the operator definition (A.21), and average over the nucleon spin. This gives

$$
f_{q}^{h}(x)=\frac{1}{4} \hat{\sum}_{n} \delta\left(p_{-} x-p_{-}+p_{n-}\right) \operatorname{Tr}\left(\left(\not p+M_{N}\right) \Gamma\left(p, p_{n}\right) \gamma^{+} \bar{\Gamma}\left(p, p_{n}\right)\right) .
$$

For the fragmentation function (A.22), we use the charge conjugation relations of the quark field operators $\mathcal{C} \psi_{\alpha} \mathcal{C}^{-1}=\left(C \gamma^{0}\right)_{\alpha \beta} \psi_{\beta}^{\dagger}, \mathcal{C} \bar{\psi}_{\alpha} \mathcal{C}^{-1}=\psi_{\beta} C_{\beta \alpha}$, 
where where $C=i \gamma^{2} \gamma^{0}$, to rewrite the matrix elements in (A.22) as follows:

$$
\begin{aligned}
& <0\left|\psi_{\alpha}\right| p, \overline{p_{n}}>=\left(C \gamma_{0}\right)_{\alpha \beta}<p_{n}, \bar{p}\left|\psi_{\beta}\right| 0>^{*} \\
& <p, \bar{p}_{n}\left|\bar{\psi}_{\alpha}\right| 0>=<\bar{p}, p_{n}\left|\psi_{\beta}\right| 0>C_{\beta \alpha} .
\end{aligned}
$$

Then we use $C \gamma^{\mu} C=\left(\gamma^{\mu}\right)^{T}$ and (A.24) to write

$<p, \bar{p}_{n}\left|\bar{\psi}_{\alpha}\right| 0>\gamma_{\alpha \beta}^{+}<0\left|\psi_{\beta}\right| p, \overline{p_{n}}>=\bar{v}_{N}(\mathbf{p} s)\left(\Gamma\left(-p, p_{n}\right) \gamma^{+} \bar{\Gamma}\left(-p, p_{n}\right)\right) v_{N}(\mathbf{p} s) \cdot 2 M_{N}$.

Averaging over the nucleon spins we get finally

$D_{q}^{h}(z)=-\frac{z}{6} \frac{1}{4} \hat{\sum}_{n} \delta\left(\frac{p_{-}}{z}-p_{-}-p_{n-}\right) \operatorname{Tr}\left(\left(-\not p+M_{N}\right) \bar{\Gamma}\left(-p, p_{n}\right) \gamma^{+} \Gamma\left(-p, p_{n}\right)\right)$.

Comparison of (A.25) and (A.28) gives

$$
D_{q}^{h}(z)=-\left.\frac{z}{6} f_{q}^{h}\left(x=\frac{1}{z}\right)\right|_{p \rightarrow-p},
$$

where $p \rightarrow-p$ means to reverse all 4 components of $p^{\mu}$, and after this replacement $p^{0}=E_{N}(\boldsymbol{p})>0$.

We now consider the property of the distribution function (A.25) under $p^{\mu} \rightarrow-p^{\mu}$. Expressing the summation $\hat{\sum}_{n}$ in terms of light cone momenta, the distribution (A.25) can be written in the form

$$
\begin{aligned}
f_{q}^{h}(x) & =\frac{1}{4} \sum_{n} \int \frac{\mathrm{d}^{4} k}{(2 \pi)^{3}} \frac{\Theta\left(p_{-}(1-x)\right)}{2 p_{-}(1-x)} \delta\left(k_{+}-e_{N}(\boldsymbol{p})+e_{n}(\boldsymbol{p}-\boldsymbol{k})\right. \\
& \times \delta\left(k_{-}-p_{-} x\right) \operatorname{Tr}\left(\left(\not p+M_{N}\right) \Gamma(p, p-k) \gamma^{+} \bar{\Gamma}(p, p-k)\right),(
\end{aligned}
$$

where $e_{n}\left(\boldsymbol{p}_{n}\right)=\frac{\boldsymbol{p}_{n \perp}^{2}+M_{n}^{2}}{2 p_{n-}}$ and $e_{N}(\boldsymbol{p})=\frac{\boldsymbol{p}_{\perp}^{2}+M_{N}^{2}}{2 p_{-}}$. We then replace $p^{\mu} \rightarrow$ $-p^{\mu}$, and then $k^{\mu} \rightarrow-k^{\mu}$ in the integral. This gives

$$
\begin{aligned}
\left.f_{q}^{h}(x)\right|_{p \rightarrow-p} & =\frac{-1}{4} \sum_{n} \int \frac{\mathrm{d}^{4} k}{(2 \pi)^{3}} \frac{\Theta\left(p_{-}(x-1)\right)}{2 p_{-}(1-x)} \delta\left(k_{+}-e_{N}(\boldsymbol{p})+e_{n}(\boldsymbol{p}-\boldsymbol{k})\right) \\
& \times \delta\left(k_{-}-p_{-} x\right) \operatorname{Tr}\left(\left(-\not p+M_{N}\right) \Gamma(-p,-p+k) \gamma^{+} \bar{\Gamma}(-p,-p+k)\right) .
\end{aligned}
$$


Because the result of taking the trace in (A.31) must be the plus component of a Lorentz four vector constructed from $p^{\mu}$ and $k^{\mu}$, we have

$$
\begin{gathered}
\operatorname{Tr}\left(\left(-\not p+M_{N}\right) \Gamma(-p,-p+k) \gamma^{+} \bar{\Gamma}(-p,-p+k)\right) \\
\quad=-\operatorname{Tr}\left(\left(\not p+M_{N}\right) \Gamma(p, p-k) \gamma^{+} \bar{\Gamma}(p, p-k)\right) .
\end{gathered}
$$

If we use (A.30) to define a function $F(x)$ by $f_{q}^{h}(x)=\Theta(1-x) F(x)$, we obtain from (A.31) and (A.32): $\left.f_{q}^{h}(x)\right|_{p \rightarrow-p}=\Theta(x-1) F(x)$. From (A.29) we then obtain the connection between the distribution and the fragmentation function as

$$
\begin{aligned}
f_{q}^{h}(x) & =\Theta(1-x) F(x) \\
D_{q}^{h}(z) & =-\Theta(1-z) \frac{z}{6} F\left(\frac{1}{z}\right) .
\end{aligned}
$$

(For spinless bosons, there is no minus sign in (A.34).) This result agrees with (A.20), and would suggest that $f_{q}^{h}$ and $D_{q}^{h}$ are essentially one and the same function, defined in different regions of the variable.

\section{B DLY transformation of evolution kernels}

In this appendix we explain the DLY based relation between the evolution kernels for distribution and fragmentation functions, which is known to be valid in LO [15].

Using Eq.(2.11), we consider the following transformation of the quark and gluon distribution functions:

$$
\begin{aligned}
& f_{q}^{h}(x) \rightarrow\left( \pm \frac{z}{6}\right) f_{q}^{h}\left(x=\frac{1}{z}\right) \\
& f_{g}^{h}(x) \rightarrow\left(\mp \frac{z}{16}\right) f_{g}^{h}\left(x=\frac{1}{z}\right)
\end{aligned}
$$


where the upper (lower) sign holds if $h$ is a boson (fermion). Using the well known evolution equations in LO [5], it is easy to derive the corresponding transformation of the evolution kernels. For the minus-type (flavor nonsinglet) combination $q-\bar{q}$, the kernel $\left(P_{q q}\right)$ is unchanged. For the flavor singlet combination $\sum_{i=1}^{N_{f}} q_{i}+\bar{q}_{i}$, which couples with to gluon, the evolution kernel is transformed as follows:

$$
\left(\begin{array}{cc}
P_{q q}(x) & P_{q g}(x) \\
P_{g q}(x) & P_{g g}(x)
\end{array}\right) \longrightarrow\left(\begin{array}{cc}
P_{q q}(z) & 2 N_{f} P_{g q}(z) \\
\frac{1}{2 N_{f}} P_{q g}(z) & P_{g g}(z)
\end{array}\right)
$$

Here $N_{f}=3$ is the number of flavors used in the $Q^{2}$ evolution equations. For reference, we summarize the forms of the individual kernels below:

$$
\begin{aligned}
P_{q q}(x) & =\frac{4}{3}\left[\frac{1+x^{2}}{(1-x)_{+}}+\frac{3}{2} \delta(x-1)\right], \\
P_{q g}(x) & =N_{f}\left[x^{2}+(1-x)^{2}\right], \\
P_{g q}(x) & =\frac{4}{3} \frac{1+(1-x)^{2}}{x}, \\
P_{g g}(x) & =6\left[\frac{x}{(1-x)_{+}}+\frac{1-x}{x}+x(1-x)\right]+\left(\frac{11}{2}-\frac{N_{f}}{3}\right) \delta(1-x) .
\end{aligned}
$$

